Western University

Scholarship@Western

$5-1-2013$

\title{
Three-dimensional segmentation of three-dimensional ultrasound carotid atherosclerosis using sparse field level sets.
}

E Ukwatta

J Yuan

D Buchanan

B Chiu

J Awad

See next page for additional authors

Follow this and additional works at: https://ir.lib.uwo.ca/biophysicspub

Part of the Medical Biophysics Commons

Citation of this paper:

Ukwatta, E; Yuan, J; Buchanan, D; Chiu, B; Awad, J; Qiu, W; Parraga, G; and Fenster, A, "Three-dimensional segmentation of three-dimensional ultrasound carotid atherosclerosis using sparse field level sets."

(2013). Medical Biophysics Publications. 125.

https://ir.lib.uwo.ca/biophysicspub/125 


\section{Authors}

E Ukwatta, J Yuan, D Buchanan, B Chiu, J Awad, W Qiu, G Parraga, and A Fenster 


\title{
Three-dimensional segmentation of three-dimensional ultrasound carotid atherosclerosis using sparse field level sets
}

\author{
E. Ukwatta ${ }^{a)}$ \\ Biomedical Engineering Graduate Program and Robarts Research Institute, The University of Western Ontario, \\ London, Ontario N6A 3K7, Canada \\ J. Yuan and D. Buchanan \\ Robarts Research Institute, The University of Western Ontario, London, Ontario N6A 3K7, Canada \\ B. Chiu \\ Department of Electronic Engineering, City University of Hong Kong, Hong Kong \\ J. Awad and W. Qiu \\ Robarts Research Institute and Department of Medical Biophysics, The University of Western Ontario, London, \\ Ontario N6A 3K7, Canada \\ G. Parraga and A. Fenster \\ Biomedical Engineering Graduate Program, Robarts Research Institute, and Department of Medical \\ Biophysics, The University of Western Ontario, London, Ontario N6A 3K7, Canada
}

(Received 26 September 2012; revised 23 February 2013; accepted for publication 25 March 2013; published 17 April 2013)

Purpose: Three-dimensional ultrasound (3DUS) vessel wall volume (VWV) provides a 3D measurement of carotid artery wall remodeling and atherosclerotic plaque and is sensitive to temporal changes of carotid plaque burden. Unfortunately, although 3DUS VWV provides many advantages compared to measurements of arterial wall thickening or plaque alone, it is still not widely used in research or clinical practice because of the inordinate amount of time required to train observers and to generate 3DUS VWV measurements. In this regard, semiautomated methods for segmentation of the carotid media-adventitia boundary (MAB) and the lumen-intima boundary (LIB) would greatly improve the time to train observers and for them to generate 3DUS VWV measurements with high reproducibility. Methods: The authors describe a 3D algorithm based on a modified sparse field level set method for segmenting the MAB and LIB of the common carotid artery (CCA) from 3DUS images. To the authors' knowledge, the proposed algorithm is the first direct 3D segmentation method, which has been validated for segmenting both the carotid MAB and the LIB from 3DUS images for the purpose of computing VWV. Initialization of the algorithm requires the observer to choose anchor points on each boundary on a set of transverse slices with a user-specified interslice distance (ISD), in which larger ISD requires fewer user interactions than smaller ISD. To address the challenges of the MAB and LIB segmentations from 3DUS images, the authors integrated regional- and boundarybased image statistics, expert initializations, and anatomically motivated boundary separation into the segmentation. The MAB is segmented by incorporating local region-based image information, image gradients, and the anchor points provided by the observer. Moreover, a local smoothness term is utilized to maintain the smooth surface of the MAB. The LIB is segmented by constraining its evolution using the already segmented surface of the $\mathrm{MAB}$, in addition to the global region-based information and the anchor points. The algorithm-generated surfaces were sliced and evaluated with respect to manual segmentations on a slice-by-slice basis using 21 3DUS images.

Results: The authors used ISD of 1, 2, 3, 4, and $10 \mathrm{~mm}$ for algorithm initialization to generate segmentation results. The algorithm-generated accuracy and intraobserver variability results are comparable to the previous methods, but with fewer user interactions. For example, for the ISD of $3 \mathrm{~mm}$, the algorithm yielded an average Dice coefficient of $94.4 \% \pm 2.2 \%$ and $90.6 \% \pm 5.0 \%$ for the MAB and LIB and the coefficient of variation of $6.8 \%$ for computing the VWV of the CCA, while requiring only $1.72 \mathrm{~min}$ (vs $8.3 \mathrm{~min}$ for manual segmentation) for a 3DUS image.

Conclusions: The proposed 3D semiautomated segmentation algorithm yielded high-accuracy and high-repeatability, while reducing the expert interaction required for initializing the algorithm than the previous 2D methods. (C) 2013 American Association of Physicists in Medicine. [http://dx.doi.org/10.1118/1.4800797]

Key words: 3D carotid segmentation, 3DUS images, carotid atherosclerosis, sparse field level set, vessel wall volume, intima-media thickness 


\section{INTRODUCTION}

Carotid atherosclerosis occurs due to the gradual accumulation of fatty deposits, macrophages, and scar tissue in the artery wall that results in the remodeling of the artery wall and the formation of atherosclerotic plaque. ${ }^{1}$ Emboli that may be generated from rupture of vulnerable plaques are the major cause of transient ischemic attacks and ischemic stroke. ${ }^{1}$ Noninvasive imaging methods such as Doppler ultrasound (US), two-dimensional ultrasound (2DUS), magnetic resonance imaging (MRI), and three-dimensional ultrasound (3DUS) have been shown to be useful for risk stratification, evaluation of the contribution of risk factors to atherosclerosis, evaluation of a patient's response to therapy, genetic research, and evaluation of efficacy of potential new therapies. ${ }^{2-12}$ 3DUS imaging provides another way to monitor plaque changes and vessel remodeling and this can be used to complement the quantification of vessel stenosis to guide and monitor medical interventions. ${ }^{5-12}$ Quantitative measurements for monitoring carotid plaque burden in longitudinal studies have been proposed previously, such as total plaque volume $e^{6,7}$ (TPV), vessel wall volume ${ }^{8-11}$ (VWV), and vessel-wall-plus-plaque-thickness (VWT) maps. ${ }^{12}$ TPV is the total plaque burden in the left or right carotid arteries measured over a specified distance. VWV is the volume enclosed between the media-adventitia boundary (MAB) and lumen-intima boundary (LIB) including plaque burden, whereas VWT is the thickness of the vessel wall, i.e., the distance between the MAB and LIB in 3D. The delineation of the carotid MAB and LIB [see Figs. 1(a) and 1(b)] are required in generating VWV and VWT measurements and are helpful in generating TPV. Although, VWV has been shown to be more sensitive to temporal changes in carotid atherosclerosis than the more widely used intima-media thickness (IMT), ${ }^{13}$ 3DUS VWV is not used because manual segmentation of the carotid MAB and LIB is tedious and timeconsuming. Currently, VWV measurements are mainly generated using manual segmentation, ${ }^{8-10}$ and there have been a few attempts ${ }^{11,14,15}$ to develop and use semiautomated algorithms. Previously developed semiautomated algorithms ${ }^{14,16}$ required user interactions on every individual slice. For example, the $2 \mathrm{D}$ method $^{15}$ required $1.6 \pm 0.3 \mathrm{~min}$ of user in- teraction time to initialize a $1 \mathrm{~cm}$ section of the common carotid artery (CCA). Therefore, the objective of this work is to develop and evaluate a 3D semiautomated algorithm to segment the MAB and LIB of the CCA from 3DUS images with minimal user interaction, while maintaining the precision, accuracy, and reproducibility of the method. Unlike the 2D methods, ${ }^{15,16}$ the proposed 3D algorithm can be initialized with ISDs greater than $1 \mathrm{~mm}$. For example, for ISDs of 2 and $3 \mathrm{~mm}$, initialization time would be approximately 0.8 and $0.53 \mathrm{~min}$, respectively.

\section{I.A. Previous studies}

Some carotid ultrasound-based segmentation techniques reported in the literature are two-dimensional and used to determine the IMT measurements in longitudinal views from carotid 2DUS images. ${ }^{17-20}$ However, for the purpose of obtaining volumetric measurements, the entire MAB and LIB surfaces need to be delineated in either transverse views or in 3D combining both transverse and longitudinal views. In this paper, we specifically focus on 2D and 3D algorithms that have been developed for segmenting the entire surface of carotid MAB and/or LIB from 3DUS images as shown in Table I. The algorithms can be divided into two main categories: Techniques ${ }^{21-26}$ that only segment the carotid LIB and ones ${ }^{14-16}$ that segment both the carotid MAB and LIB. For example, Gill et al. ${ }^{25}$ and Solovey ${ }^{26}$ proposed direct 3D segmentation methods only for LIB segmentation. Gill et al. ${ }^{25}$ used a 3D dynamic balloon model that is inflated to coarsely locate the boundary and then refined using edge-based energies for LIB segmentation. However, edgebased segmentation alone is not adequate to stop leaking at low-contrast boundaries in US images. ${ }^{28}$ Solovey ${ }^{26}$ proposed a level set-based method to segment the LIB by incorporating a region term that minimizes the probability distribution overlap and a weak geometric prior, which encourages convexity of the boundary in a level set framework. However, the carotid LIB may not have a convex shape due to the presence of plaque, which is most prominent close to the bifurcation, as shown in Fig. 2. Another limitation of some of the studies ${ }^{21-23}$ for LIB segmentation is that they validated their algorithm on only seven 2D B-mode patient images, ${ }^{21}$

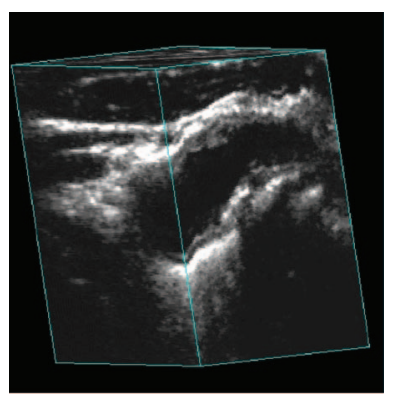

(a)

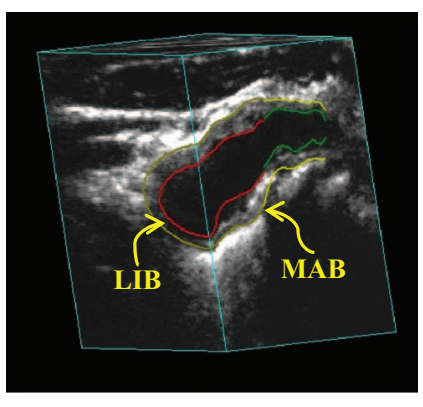

(b)

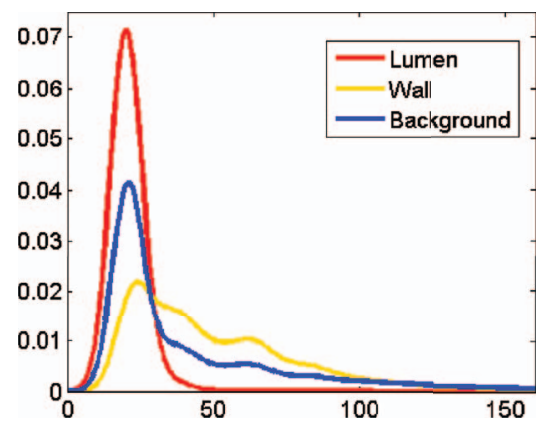

(c)

FIG. 1. (a) An example 3D carotid US image. (b) 3D carotid US image with overlaid user-drawn manual contours of the MAB and LIB. Lumen is enclosed within the LIB and vessel wall is enclosed in between the MAB and LIB. (c) Intensity probability density functions (PDF) for lumen, wall, and background regions for the example 3DUS image. Note that the intensity PDFs have high overlap with each other. 
TABLE I. Previous papers describing carotid LIB and/or MAB segmentations from 3DUS/B-mode images.

\begin{tabular}{|c|c|c|c|c|c|c|c|}
\hline Paper & Year & Dimension & LIB & MAB & Time (min) & No. of images (type) & Other information \\
\hline $\begin{array}{l}\text { Ukwatta } \\
\text { (Ref. 16) }\end{array}$ & 2011 & $2 \mathrm{D}$ & $\begin{array}{l}\text { Coupled level } \\
\text { set }\end{array}$ & $\begin{array}{l}\text { Coupled level } \\
\text { set }\end{array}$ & $3.7 \pm 0.4$ & 21 (3DUS) & $\begin{array}{l}\text {-Anchor points on the } \\
\text { boundary on each slice. }\end{array}$ \\
\hline $\begin{array}{l}\text { Ukwatta } \\
\text { (Ref. 15) }\end{array}$ & 2011 & $2 \mathrm{D}$ & Level set & Level set & $2.8 \pm 0.4$ & 21 (3DUS) & $\begin{array}{l}\text {-Anchor points on the } \\
\text { boundary on each slice. }\end{array}$ \\
\hline Yang (Ref. 14) & 2011 & $2 \mathrm{D}$ & $\begin{array}{l}\text { Active shape } \\
\text { model }\end{array}$ & $\begin{array}{l}\text { Active shape } \\
\text { model }\end{array}$ & $4.4 \pm 0.6$ & 68 (3DUS) & $\begin{array}{l}\text {-Initializing a shape model } \\
\text { on each slice. } \\
\text {-Requires training data. }\end{array}$ \\
\hline $\begin{array}{l}\text { Solovey } \\
\text { (Ref. 26) }\end{array}$ & 2010 & $3 \mathrm{D}$ & Level set & - & - & 2 (3DUS) & $\begin{array}{l}\text {-Seed points inside the } \\
\text { lumen. }\end{array}$ \\
\hline Gill (Ref. 25) & 2000 & $3 \mathrm{D}$ & $\begin{array}{l}\text { Dynamic } \\
\text { balloon model }\end{array}$ & - & $30 \mathrm{~s}$ & 1 (3DUS) & $\begin{array}{l}\text {-Initializing the balloon } \\
\text { model inside the LIB. }\end{array}$ \\
\hline $\begin{array}{l}\text { Guerrero } \\
\text { (Ref. 24) }\end{array}$ & 2007 & $2 \mathrm{D}$ & $\begin{array}{l}\text { Star Kalman } \\
\text { filters }\end{array}$ & - & $23 \mathrm{~ms}$ & 3 (B-mode sequence) & -Seed point inside the LIB. \\
\hline $\begin{array}{l}\text { Abolmaesumi } \\
\text { (Ref. 23) }\end{array}$ & 2000 & $2 \mathrm{D}$ & $\begin{array}{l}\text { Star algorithm } \\
\text { /Kalman } \\
\text { filtering }\end{array}$ & - & - & 1 (B-mode sequence) & $\begin{array}{l}\text {-Seed point inside the LIB. } \\
\text {-Quantitative Results not } \\
\text { reported. }\end{array}$ \\
\hline $\begin{array}{l}\text { Zahalka } \\
\text { (Ref. 22) }\end{array}$ & 2001 & $2 \mathrm{D}$ & $\begin{array}{l}\text { Active } \\
\text { contours }\end{array}$ & - & $25 \mathrm{~s} /$ slice & 2 (3DUS) & $\begin{array}{l}\text {-Seed point inside the LIB } \\
\text {-Results not reported. }\end{array}$ \\
\hline Mao (Ref. 21) & 2000 & $2 \mathrm{D}$ & $\begin{array}{l}\text { Active } \\
\text { contours }\end{array}$ & - & $0.8 \mathrm{~s} /$ slice & 7 (B-mode) & -Seed point inside the LIB \\
\hline
\end{tabular}

few phantom images, ${ }^{22}$ or did not report on a quantitative segmentation validation. ${ }^{23}$ There have been three previous methods that segment both the carotid MAB and LIB from 3DUS images. ${ }^{14-16}$ Yang et al. ${ }^{14}$ used active shape models to segment the carotid LIB and MAB slicewise, which involved a large number of training images to generate models. However, the shape of the LIB could be different between subjects, which is also affected by the degree of stenosis, as shown in Fig. 2. Ukwatta et al. ${ }^{15}$ proposed a 2D segmentation method for delineating the carotid arteries on a slice-by-slice basis on transverse slices extracted from 3DUS images. Ukwatta et al. ${ }^{16}$ used a coupled level set approach to simultaneously segment the MAB and LIB. For both techniques, the areas from individual segmentations are summed together to generate the VWV. Since each slice is segmented independently at an interslice distance (ISD) of $1 \mathrm{~mm}$, the 2D methods require user interaction on every slice. Although the slice thickness of our 3DUS images is approximately $0.15 \mathrm{~mm}$, the $2 \mathrm{D}$ methods ${ }^{15,16}$ make an assumption that there
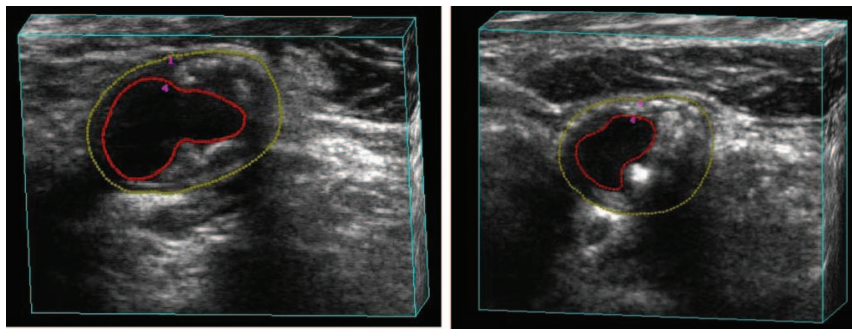

FIG. 2. Two example transverse views of the CCA with overlaid manual segmentations of the MAB and LIB from 3DUS images of two subjects with carotid stenosis of more than $60 \%$. Note that although the MAB has a relatively low order convex shape, the LIB has a nonconvex shape. are no considerable changes in the vessel morphology within an ISD of $1 \mathrm{~mm}$.

\section{I.B. Contributions}

In this paper, we describe and evaluate a direct $3 \mathrm{D}$ segmentation algorithm to delineate the MAB and LIB of the carotid CCA from 3DUS images. The algorithm uses a similar initialization approach used previously for the $2 \mathrm{D}$ segmentation, ${ }^{15}$ but requires initialization only on a smaller subset of transverse slices than used in the $2 \mathrm{D}$ method. ${ }^{15}$ However, the proposed method does not hold the assumption of constant vessel morphology within an ISD of $1 \mathrm{~mm}$, as it provides a direct 3D segmentation of the entire 3DUS image at a slice thickness of $0.15 \mathrm{~mm}$. The $3 \mathrm{D}$ method uses nine energy functionals in total, out of which eight were used in the $2 \mathrm{D}$ method. ${ }^{15}$ However, the main contribution of this paper is the extension of the energy functions ${ }^{15}$ to $3 \mathrm{D}$. To the best of our knowledge, the proposed algorithm is the first direct 3D segmentation method for segmenting both the carotid MAB and LIB from 3DUS images for the purpose of computing the VWV. A direct 3D segmentation method has the potential for reducing user interaction over a $2 \mathrm{D}$ method, while increasing the robustness of the segmentation by integrating out-of-plane image information.

A preliminary study of this work has been previously described in a conference paper. ${ }^{27}$ The algorithm was briefly described and validated only for accuracy using 15 3DUS images in the conference paper. ${ }^{27}$ In this paper, we provide comprehensive details of the proposed algorithm and provide a thorough analysis of the accuracy and intraobserver variability on 21 3DUS images with five repeated algorithm segmentations. In addition, initialization of the algorithm is 

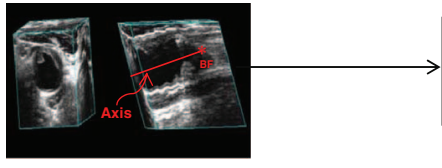

Algorithm Initialization With a Predetermined ISD

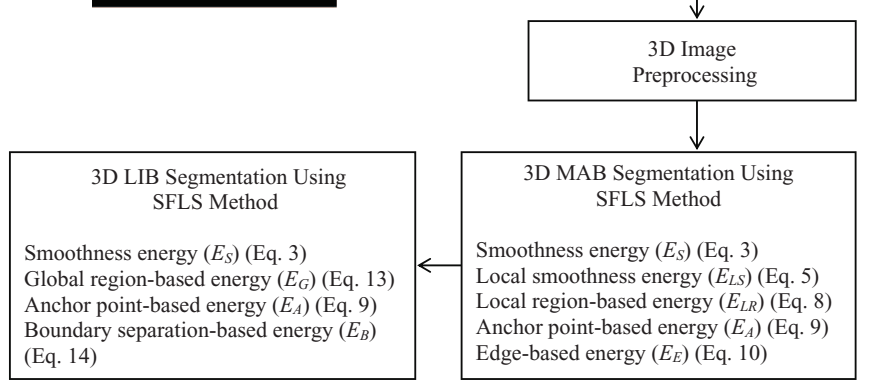

FIG. 3. Block diagram of the workflow of the algorithm.

investigated with respect to accuracy and variability with ISD of $1,2,3,4$, and $10 \mathrm{~mm}$.

\section{MATERIALS AND METHODS}

Our segmentation pipeline consists of four main steps as shown in Fig. 3: Algorithm initialization, 3D image preprocessing, MAB segmentation, and LIB segmentation. Each of the steps is described below.

\section{II.A. Algorithm initialization}

A boundary-based initialization approach is used in our algorithm as opposed to a region-based initialization, where the observer chooses "anchor points" on the MAB and LIB. The anchor points are used to generate the initial mask for the segmentation and constrain the evolving surface to always pass through them.

At the point of the bifurcation, the common carotid artery divides into the internal and external carotid arteries [see Fig. 1(a) of Ukwatta et al. ${ }^{15}$ ]. The observer first locates the bifurcation point (BF) and defines the long axis of the artery as shown in Fig. 3. Then, the observer chooses four anchor points each on the MAB and LIB on a set of transverse slices, which are orthogonal to the long axis of the artery, with a predefined ISD. For example, ISD of 1, 2, 3, 4, and $10 \mathrm{~mm}$ are used in this paper, where large ISDs result in fewer user interactions. In comparison to the $2 \mathrm{D}$ method, ${ }^{15}$ which requires user initializations on every slice at an ISD of $1 \mathrm{~mm}$, our 3D method uses fewer slices for intialization. Based on the evaluation results, ISDs of 2 and $3 \mathrm{~mm}$ are suitable for initialization without compromising the accuracy, thus the number of slices for initialization is reduced by a factor of 2 or 3 .

Figure 4 shows the steps for generating a 3D mask to represent the initial surface for the segmentation algorithm for the LIB or MAB. For the set of 2D planes with user-initialized anchor points, $2 \mathrm{D}$ contours are first generated by cubic spline interpolation with approximately $0.2 \mathrm{~mm}$ interval between adjacent points using the four anchor points on each plane. The 2D contours are then corrected for any intersections of the MAB and LIB automatically, by correcting the LIB to have a minimum separation of $0.5 \mathrm{~mm}$ from the carotid MAB ${ }^{29}$ The contour points between two adjacent parallel slices are then corresponded using a symmetric correspondence algorithm. ${ }^{30}$ The symmetric correspondence algorithm provides an improvement to the closest point algorithm by avoiding multiple points mapped to the same point. ${ }^{30} \mathrm{~A}$ triangular surface is generated using the corresponding points, which is then converted to a 3D binary mask. Any voxel inside the triangular surface is assigned label one and any voxel outside the surface is labeled zero.

\section{II.B. 3D image preprocessing}

The preprocessing steps of our algorithm are shown in Fig. 5. Initially, a region-of-interest (ROI) is chosen by using a $7 \mathrm{~mm}$ margin in all three directions from the minimum and maximum values of $\mathrm{x}, \mathrm{y}$, and $\mathrm{z}$ coordinates of the initial surface. Two separate preprocessing pipelines are used for the MAB and LIB as shown in Fig. 5. The 3DUS image is first normalized using the minimum and maximum intensity of the image to reduce the effect of different image contrast of each image. An edge-preserving anisotropic diffusion filtering ${ }^{31}$ is then applied to reduce the effect of image speckle, as shown in previous studies. ${ }^{28,32,34}$ This method enhances the 3DUS image by diffusing image regions with low or no image gradient while preserving image edges. In addition, it provides the filtered output image within a few seconds for 3DUS images. The output from the diffusion filtering is used as the input 3DUS image for LIB segmentation. For MAB segmentation, filtered output from the Gaussian filter with a kernel size of $0.7 \mathrm{~mm}$ is used as the input 3DUS image. The rest of the preprocessing steps are applied to obtain an edge map to aid in
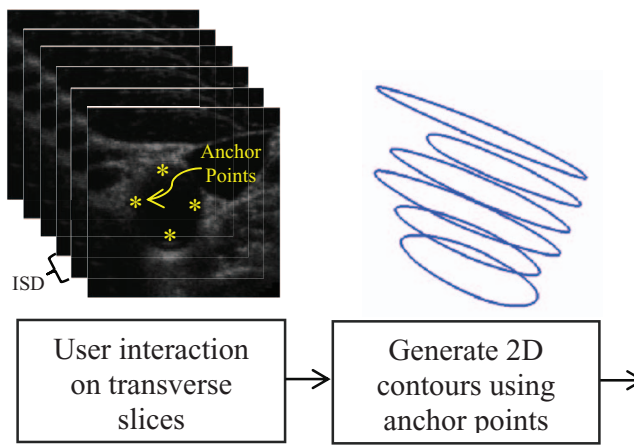

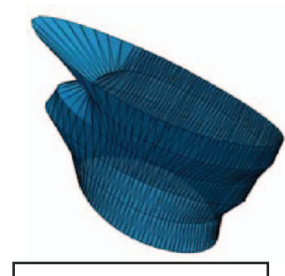

Generate triangulated surface

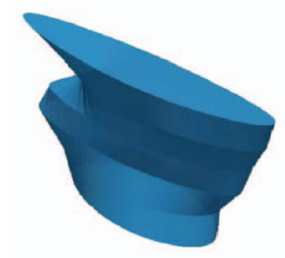

Generate a 3D mask for algorithm initialization

FIG. 4. The process of creating a 3D mask from anchor points as the initial surface for the algorithm with an ISD of $2 \mathrm{~mm}$. 


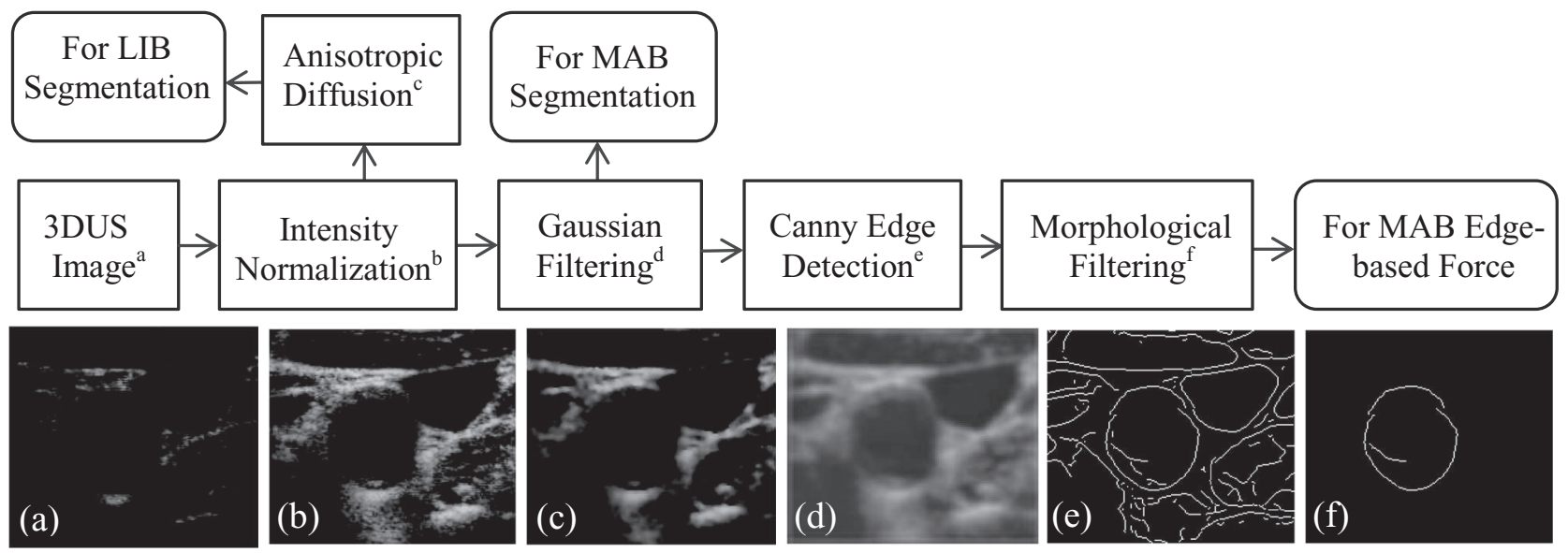

FIG. 5. 3D image preprocessing steps of the algorithm. The results here are shown for a single slice of the 3DUS image.

the MAB segmentation. We apply the Canny edge detector ${ }^{33}$ on the filtered 3DUS images to obtain an edge map, as previously used in US segmentation tasks. ${ }^{34}$ Morphological operations are then used on the edge map to remove edge segments that are smaller than $2 \mathrm{~mm}$, and edges that are more than a distance of $10 \mathrm{~mm}$ from the initial surface. The resulting filtered edge map is incorporated into the objective function of MAB segmentation. In this paper, values for image preprocessing parameters are determined via a systematic search of the space of parameter values. The parameters and their optimized values for preprocessing are shown in Table II.

\section{II.C. Sparse field level set (SFLS) method}

In this paper, we used a SFLS implementation proposed by Whitaker et al. ${ }^{35}$ for both LIB and MAB segmentations. The SFLS method updates only the level set evolution around the neighborhood of zero level set rather than computing $\phi(\mathbf{x})$ for the whole image domain, to speed up the evolution by reducing the amount of computation. Let the bounded open subset $\Omega \subset \mathbb{R}^{3}$ represent the image domain, where the image $I: \Omega \rightarrow \mathbb{R}$ and $\mathbf{x} \in \Omega$ is a spatial variable. The level set is an implicit representation of a surface defined by the Lipschitz function $\phi(\mathbf{x}): \Omega \rightarrow \mathbb{R}$, where the zero level of $\phi(\mathbf{x})$ represents the segmented surface. $\phi(\mathbf{x})$ is represented as a signed distance function at the beginning, where $\phi(\mathbf{x})<0$ inside the surface. The level set evolution equation is given by

$$
\frac{\partial \phi(\mathbf{x})}{\partial t}+\nabla E|\nabla \phi(\mathbf{x})|=0
$$

where $E$ is the objective function representing the goal of segmentation. ${ }^{36,37} \phi(\mathbf{x})$ is evolved based on the objective function, until it converges to an energy minimum. The objective function contains two types of energy terms: external energy terms, derived from the (preprocessed) image data, and internal energy terms, derived from high-level knowledge about the expected shape of the boundaries as well as from user-specified anchor points.

\section{II.D. MAB segmentation}

At first, we segment the MAB using the 3D SFLS method. The segmentation task of the MAB from 3DUS carotid images is challenging due to the following reasons: Low image contrast of the MAB especially on the surface that is parallel to the US beam, presence of calcified plaque creating shadowing and signal dropouts, heterogeneous intensities on the interior and exterior of the MAB (contain both hypoechoic and hyperechoic regions), and overlapping intensity probability density functions (PDF) with each other as shown in Fig. 1(c). To address these challenges in the segmentation, we incorporated five types of energy criteria into the objective functional ( $\left.E_{\mathrm{MAB}}\right)$ for the MAB (see Fig. 3):

$$
E_{\mathrm{MAB}}=\alpha_{S} E_{S}+\alpha_{L S} E_{L S}+\alpha_{L R} E_{L R}+\alpha_{A} E_{A}+\alpha_{E} E_{E},
$$

where weights $\alpha_{S}, \alpha_{L S}, \alpha_{L R}, \alpha_{A}, \alpha_{E}$ are positive constants.

The smoothness energy ${ }^{37}$ [Eq. (3)] is the standard regularization used in level sets that penalizes the length of the

TABLE II. Parameters and their optimized values for the preprocessing.

\begin{tabular}{lll}
\hline \hline Operation & \multicolumn{1}{c}{ Parameters } & \multicolumn{1}{c}{ Values } \\
\hline Diffusion filtering & No. of iterations, time step, conductance & $4,0.2,1.0$ \\
Gaussian filter & Kernel size, SD & $0.7,0.3 \mathrm{~mm}$ \\
Canny edge filter & High and low threshold & $0.25,0.08$ \\
Morphological opening & Element size, minimum size maximum distance & $2.5,2,10,2 \mathrm{~mm}$ \\
\hline \hline
\end{tabular}


segmentation surface and is given by

$$
E_{S}=\int_{\Omega}|\nabla H(\phi(\mathbf{x}))| d \mathbf{x},
$$

where

$$
H(x)=\left\{\begin{array}{cl}
1 & \text { if } x<-\varepsilon \\
0 & \text { if } x>\varepsilon \\
\frac{1}{2}\left[1+\frac{x}{\varepsilon}+\frac{1}{\pi} \sin \left(\frac{\pi x}{\varepsilon}\right)\right] & \text { if }|x| \leq \varepsilon,
\end{array}\right.
$$

is the regularized Heaviside function ${ }^{37,39}$ representing interior of the segmentation surface, whereas the exterior region of the surface is defined as $(1-H(\phi(\mathbf{x})) . \varepsilon$ is a small positive constant.

The local smoothness energy ${ }^{38}\left(E_{L S}\right)$ reduces the convexity and concavity of a local neighborhood region defined by a spherical mask. Let $\hat{\mathbf{x}} \in \Omega$ be another independent spatial variable:

$$
E_{L S}=\int_{\Omega} \delta(\phi(\mathbf{x}))\left(A_{v}(\mathbf{x})-A_{u}(\mathbf{x})\right)^{2} d \mathbf{x}
$$

where

$$
B_{L}(\mathbf{x}, \hat{\mathbf{x}})=\left\{\begin{array}{lc}
1 & \text { if }\|\mathbf{x}-\hat{\mathbf{x}}\|<r_{L} \\
0 & \text { otherwise }
\end{array}\right.
$$

is used to define a spherical region with localizing radius $\quad r_{L} . \quad A_{u}(\mathbf{x})=\int_{\Omega} B_{L}(\mathbf{x}, \hat{\mathbf{x}}) H(\phi(\hat{\mathbf{x}})) d \hat{\mathbf{x}} \quad$ and $\quad A_{v}(\mathbf{x})$ $=\int_{\Omega} B_{L}(\mathbf{x}, \hat{\mathbf{x}})(1-H(\phi(\hat{\mathbf{x}}))) d \hat{\mathbf{x}}$ are the volumes of the inside and outside regions of the $B_{L}(\mathbf{x}, \hat{\mathbf{x}})$ :

$$
\delta(x)=\left\{\begin{array}{cl}
1 & \text { if } x=0 \\
0 & \text { if }|x|>\varepsilon \\
\frac{1}{2 \varepsilon}\left[1+\cos \left(\frac{\pi x}{\varepsilon}\right)\right] & \text { if }|x| \leq \varepsilon
\end{array}\right.
$$

is the regularized Dirac delta function, which is the derivative of $H(x)$.

The local region-based energy ${ }^{39}\left(E_{L R}\right)$ as given by Eqs. (4) and (12) in Lankton et al. ${ }^{39}$ minimizes the intensity variance of the local interior and exterior regions:

$$
\begin{aligned}
E_{L R}= & \int_{\Omega} \delta(\phi(\mathbf{x})) \int_{\Omega} B_{L}(\mathbf{x}, \hat{\mathbf{x}})\left[H(\phi(\hat{\mathbf{x}}))(I(\hat{\mathbf{x}})-u(\mathbf{x}))^{2}\right. \\
& \left.+(1-H(\phi(\hat{\mathbf{x}})))(I(\hat{\mathbf{x}})-v(\mathbf{x}))^{2}\right] d \hat{\mathbf{x}} d \mathbf{x},
\end{aligned}
$$

where $u(x)$ and $v(\mathbf{x})$ are the mean image intensities of the interior and exterior of the active contour within the local region defined by $B_{L}(\mathbf{x}, \hat{\mathbf{x}}) .{ }^{39}$ The parameter $r_{L}$ determines the degree of blending local statistics around the boundary to global statistics of the image. A small $r_{L}$ would act as an edge detector, while an increasing $r_{L}$ would cause convergence to global statistics.

The anchor point-energy ${ }^{15}\left(E_{A}\right)$ encourages the contour to pass through each anchor point $\mathbf{x}_{A}^{i}$, placed by the observer if the evolving contour is within a distance $r_{A}$ to the anchor point:

$$
E_{A}=\sum_{i=1}^{N_{P}} \int_{\Omega} \delta(\phi(\mathbf{x})) B_{A}^{i}(\mathbf{x})\left(\phi(\mathbf{x})-\phi\left(\mathbf{x}_{A}^{i}\right)\right)^{2} d \mathbf{x},
$$

where $B_{A}^{i}(\mathbf{x})=\left\{\begin{array}{cc}1 & \text { if }\left\|\mathbf{x}-\mathbf{x}_{A}^{i}\right\|<r_{A} \\ 0 & \text { otherwise }\end{array}\right.$ is used to define a spherical mask around the anchor point with radius $r_{A}$ and $N_{P}$ is the number of anchor points. When the contour points are away from the anchor point by more than $r_{A}$ there is no influence of this energy term. In our implementation, the segmentation is started with the initial mask that already passes through the anchor points, where $E_{A}$ would be already at its minimum. With initializing the segmentation close to the MAB using the anchor points, we reduce the possibility of the evolving contour to be trapped in local minima due to the heterogeneity of the region and also reduce the computational time for segmentation.

The edge-based level set framework was initially proposed by Caselles et al. ${ }^{40}$ They proposed a term, which integrates a function of the image gradient along the boundary, as given by the second term of Eq. (3). ${ }^{40}$ We use a modified edge-based energy $^{15}$ term $\left(E_{E}\right)$ to complement the region-based forces, especially at locations of the surface that are orthogonal to the US beam:

$$
E_{E}=-\int_{\Omega} \delta(\phi(\mathbf{x})) \frac{B_{E}(\mathbf{x})}{\varepsilon+\left|\phi(\mathbf{x})-\phi\left(X_{E}(\mathbf{x})\right)\right|} d \mathbf{x},
$$

where $B_{E}(\mathbf{x})=\left\{\begin{array}{cc}1 & \text { if } 0<\left\|\mathbf{x}-X_{E}(\mathbf{x})\right\|<r_{E} \\ 0 & \text { otherwise }\end{array}\right.$ is the spherical region around the point $\mathbf{x}$ with a radius $r_{E}$ and $X_{E}(\mathbf{x})$ $=\underset{\hat{\mathbf{x}} \in s}{\arg \min }\|\mathbf{x}-\hat{\mathbf{x}}\|, s=\{\hat{\mathbf{x}} ; M(\hat{\mathbf{x}})=1\}$ being the nearest edge point from point $\mathbf{x}$ and $M(\mathbf{x})=\left\{\begin{array}{ll}1 & \text { if } \mathbf{x} \text { lies on an edge } \\ 0 & \text { otherwise }\end{array}\right.$ is the edge map obtained from preprocessing. The edge force is nonzero when the contour is within a distance $r_{E}$ to the closest edge point and minimum when the contour is placed on the edge.

By taking the first variation of the $E_{\mathrm{MAB}}[\mathrm{Eq}$. (2)] with respect to $\phi$, we obtain the evolving equation function for the MAB segmentation where $\operatorname{sgn}(\mathbf{x})$ is the signum function: ${ }^{15}$

$$
\begin{aligned}
\frac{\partial \phi(\mathbf{x})}{\partial t}= & \delta(\phi(\mathbf{x}))\left\{\alpha_{S} \operatorname{div}\left(\frac{\nabla \phi(\mathbf{x})}{|\nabla \phi(\mathbf{x})|}\right)\right. \\
& +\alpha_{L S}\left(A_{v}(\mathbf{x})-A_{u}(\mathbf{x})\right) \\
& +\alpha_{L R} \int_{\Omega} B_{L}(\mathbf{x}, \hat{\mathbf{x}}) \delta(\phi(\hat{\mathbf{x}}))\left[(I(\hat{\mathbf{x}})-u(\mathbf{x}))^{2}\right. \\
& \left.-(I(\hat{\mathbf{x}})-v(\mathbf{x}))^{2}\right] d \hat{\mathbf{x}} \\
& +\alpha_{A} \sum_{i=1}^{N_{P}} B_{A}^{i}(\mathbf{x})\left(\phi(\mathbf{x})-\phi\left(x_{A}^{i}\right)\right) \\
& \left.+\alpha_{E} B_{E}(\mathbf{x}) \frac{\operatorname{sgn}\left(\phi(\mathbf{x})-\phi\left(X_{E}(\mathbf{x})\right)\right)}{\left(\varepsilon+\mid \phi(\mathbf{x})-\phi\left(X_{E}(\mathbf{x}) \mid\right)^{2}\right.}\right\} .
\end{aligned}
$$

\section{II.E. LIB segmentation}

After the MAB has been segmented, LIB segmentation is performed also using the 3D SFLS method. The challenges in the LIB segmentation are its low contrast image boundary and its irregular shape in some subjects due to the presence of plaque. For LIB segmentation, we use a global region-based 
energy, high-level knowledge provided by the observer, and previously obtained MAB segmentation to constrain the LIB. The objective functional $\left(E_{\mathrm{LIB}}\right)$ for the LIB comprises of four energies (see Fig. 3):

$$
E_{\mathrm{LIB}}=\lambda_{S} E_{S}+\lambda_{A} E_{A}+\lambda_{G} E_{G}+\lambda_{B} E_{B},
$$

where weights $\lambda_{S}, \lambda_{A}, \lambda_{G}, \lambda_{B}$ are positive constants. $E_{S}$ is the smoothness energy [Eq. (3)] and $E_{A}$ is the anchor point-based energy [Eq. (9)] as defined previously.

The global region-based energy ${ }^{37}$

$$
\begin{aligned}
E_{G}= & \int_{\Omega} H(\phi(\mathbf{x}))\left(I(\mathbf{x})-u_{m}\right)^{2} \\
& +(1-H(\phi(\mathbf{x})))\left(I(\mathbf{x})-v_{m}\right)^{2} d \mathbf{x}
\end{aligned}
$$

partitions the image into different regions based on their global distinct characteristics of the image region. $u_{m}$ and $v_{m}$ are the mean intensities of interior and exterior regions of the boundary. ${ }^{15,37}$ The use of a combination of local region-based and global region-based forces to segment the LIB is motivated by the fact that the intensity of the interior of the lumen is fairly homogeneous while the intensity of the exterior of the lumen is heterogeneous.

Because the MAB and LIB are separated by the intimamedia layer, we constrained them to have a minimum distance $\left(d_{B}\right)$ of $0.5 \mathrm{~mm}$ from each other. ${ }^{29}$ The algorithm-generated $\mathrm{MAB}$ is used to reduce leaking of the LIB at a low image contrast location. We imposed $d_{B}$ as a distance-based constraint to drive the LIB segmentation using the algorithm-generated MAB segmentation. The boundary separation-based energy $\left(E_{B}\right)$ is given by

$$
E_{B}=\int_{\Omega} \delta(\phi(\mathbf{x})) B_{B}(\mathbf{x}) d \mathbf{x},
$$

where $B_{B}(\mathbf{x})=\left\{\begin{array}{lc}1 & \text { if } \min _{\hat{\mathbf{x}}} D(\mathbf{x}, \hat{\mathbf{x}})<d_{B} \\ 0 & \text { otherwise }\end{array}\right.$ and $D(\mathbf{x}, \hat{\mathbf{x}})=\|\mathbf{x}-\hat{\mathbf{x}}\|$, $\nabla H\left(\phi_{\mathrm{MAB}}(\hat{\mathbf{x}})\right)=1$. The term $E_{B}$ discourages the intersection of the MAB and LIB. This energy is nonzero when the LIB moves closer to the MAB than distance $d_{B}$.

By taking the first variation of the $E_{\mathrm{LIB}}$ [Eq. (12)] with respect to $\phi$ we obtain the evolving equation for the LIB seg- mentation:

$$
\begin{aligned}
\frac{\partial \phi(\mathbf{x})}{\partial t}= & \delta(\phi(\mathbf{x}))\left\{\lambda_{S} \operatorname{div}\left(\frac{\nabla \phi(\mathbf{x})}{|\nabla \phi(\mathbf{x})|}\right)\right. \\
& +\lambda_{G}\left(\left(I(\mathbf{x})-u_{m}\right)^{2}-\left(I(\mathbf{x})-v_{m}\right)^{2}\right) \\
& \left.+\lambda_{A} \sum_{i=1}^{N_{P}} B_{A}^{i}(\mathbf{x})\left(\phi(\mathbf{x})-\phi\left(x_{A}^{i}\right)\right)+\lambda_{B} B_{B}(\mathbf{x})\right\} .
\end{aligned}
$$

The stopping criteria for MAB and LIB segmentation is based on the convergence test that terminates when the evolution does not change within bounds of $0.4 \mathrm{~mm}^{3}$ on successive iterations and a maximum iteration criterion, which is set to be 250 iterations.

Initially, the parameter values were chosen empirically, which were then optimized for the MAB and LIB segmentations using Dice similarity coefficients ${ }^{41}$ (DSC). The optimization was performed by changing a single parameter over a range of possible values, while holding the other parameters fixed for ten 3DUS images. We used DSC as the metric for parameter optimization, as it is a region-based metric that depends on spatial overlap, which is thus more stringent than volume metrics. This procedure was repeated until either convergence or a maximum of five repetitions were met. It should be noted that we performed only a local optimum search, thus the global optimum of the parameter values is not guaranteed. Table III shows the parameter values for the MAB and LIB segmentations after optimization. The parameter values were kept constant during the validation experiments.

\section{II.F. Validation}

The algorithm was evaluated for accuracy and reproducibility by comparison to user-drawn contours. Manual segmentations of the MAB and LIB were performed using 3D multiplanar viewing software ${ }^{42}$ on transverse views up to a distance of $1 \mathrm{~cm}$ of the artery from the bifurcation with an ISD of $1 \mathrm{~mm}$. For further information about the manual segmentations the readers are referred to Egger et al. ${ }^{8}$ The images were first randomized to the observer for both repeated

TABLE III. Parameters and their optimized values for the MAB and LIB segmentations.

\begin{tabular}{lccccc}
\hline \hline & \multicolumn{2}{c}{ MAB } & & \multicolumn{2}{c}{ LIB } \\
\cline { 2 - 3 } \cline { 5 - 6 } Description & Parameter & Value & & Parameter & Value \\
\hline Local region radius & $r_{L}$ & $0.7 \mathrm{~mm}$ & & - & - \\
Anchor radius & $r_{A}$ & $0.8 \mathrm{~mm}$ & & $r_{A}$ & $0.9 \mathrm{~mm}$ \\
Edge radius & $r_{E}$ & $0.8 \mathrm{~mm}$ & & - & - \\
& $\varepsilon$ & $10^{-5}$ & & & \\
Smoothness $\left(E_{S}\right)$ weight & $\alpha_{S}$ & 0.4 & & $\lambda_{S}$ & 0.6 \\
Local smoothness $\left(E_{L S}\right)$ weight & $\alpha_{L S}$ & 0.8 & & - & - \\
Local region $\left(E_{L R}\right)$ weight & $\alpha_{L R}$ & 3.1 & & - & - \\
Anchor $\left(E_{A}\right)$ weight & $\alpha_{A}$ & 1.9 & & $\lambda_{A}$ & 1.8 \\
Edge $\left(E_{E}\right)$ weight & $\alpha_{E}$ & 0.4 & & - & - \\
Global region $\left(E_{G}\right)$ weight & - & - & & $\lambda_{G}$ & 0.7 \\
Boundary-separation $\left(E_{B}\right)$ weight & - & - & & $\lambda_{B}$ & 1.9 \\
\hline \hline
\end{tabular}


manual and algorithm segmentation rounds. An observer outlined the carotid MAB and LIB five times repeatedly with a $24 \mathrm{~h}$ period between repetitions on 21 3DUS images. The observer initialized the anchor points on the same image planes as for the manual segmentation to ensure that the algorithm segmented the same 2D slices with an ISD of $1 \mathrm{~mm}$ as segmented manually for direct comparison. For the five repeated algorithm segmentations, four anchor points were chosen for both the MAB and LIB on each slice for each 3DUS image for each round with a $24 \mathrm{~h}$ period between repetitions on the same dataset as described by Ukwatta et al. ${ }^{15}$

We also evaluated the algorithm accuracy and reproducibility with different ISDs for initialization on the same dataset. ISD of $1,2,3,4$, and $10 \mathrm{~mm}$ were used in the experiments, where larger ISDs require fewer user interactions. For example, for an ISD of $2 \mathrm{~mm}$, user initializations on every other slice are used as anchor points to generate initial mask.

\section{II.F.1. Evaluation metrics}

The algorithm-generated surfaces were resliced at $1 \mathrm{~mm}$ intervals, for a direct comparison to manual segmentation. Similar to the 2D method, ${ }^{15}$ we used the same volume-based, region-based, and distance-based metrics to evaluate the accuracy of our segmentation method. Volume-based metrics capture the aspect of the segmentation that is of greatest clinical interest: the VWV. Region- and boundary distance-based metrics describe in greater detail the spatial fidelity of the segmentations to those generated manually by the observer.

The volumes were computed by multiplying the area enclosed by the boundary with the ISD. We used volume error ${ }^{15}$ [Eq. (19)] of Ukwatta et al. ${ }^{15}(\delta \mathrm{V})$ and absolute volume error $(|\delta V|)$ as the volume-based metrics. We computed $\delta V$ and $|\delta V|$ for the MAB ( $\delta V_{\mathrm{MAB}}$ and $\left.\left|\delta V_{\mathrm{MAB}}\right|\right)$, LIB ( $\delta V_{\mathrm{LIB}}$ and $\left.\left|\delta V_{\mathrm{LIB}}\right|\right)$, and $\mathrm{VWV}\left(\delta V_{\mathrm{VWV}}\right.$ and $\left.\left|\delta V_{\mathrm{VWV}}\right|\right)$. The overall mean volume error for the 3DUS image set is obtained by averaging the $\delta V$, whereas the overall absolute mean volume error is considered as the geometric mean of the $|\delta V|$.

We used coefficient of variation (CV) to compare the reproducibility of the algorithm and manual method for the generation of VWV. We also computed the minimal detectable difference (MDD) (Ref. 43) of VWV for the algorithm and manual segmentation. The MDD is used to determine the change in values between two successive measurements of VWV that can be detected at a given confidence level:

$$
\operatorname{MDD}=\mathrm{z}_{\alpha / 2} \sqrt{2} \text { SEM, }
$$

where $z_{\alpha / 2}$ is the $z$-statistic at which the difference between the two measurements has a chance of being $5 \%$ (i.e., $z_{\alpha / 2}$ $=1.96$ ) and SEM is the standard error of measurement. ${ }^{43}$

We computed average boundaries from repeated segmentations and used them for computing region-based and distance-based metrics. ${ }^{15,44}$ The DSC (Ref. 41) was used as a region-based measure to compare two segmentations for accuracy on slice-by-slice basis. We compute the mean absolute distance error ${ }^{15}$ (MAD) and maximum absolute distance error $^{15}$ (MAXD) as boundary distance-based metrics. Bound- ary distance-based metrics depend on the establishment of a pointwise correspondence between the boundaries. We used the symmetric correspondence method ${ }^{12,30}$ to establish correspondence between points on the boundaries.

\section{II.G. Study subjects and imaging}

To our knowledge, public datasets with manual segmentation as ground truth are not available for 3D carotid US images for algorithm evaluation. Therefore, we used a dataset acquired in our laboratory. The dataset used in this study has been previously described in Ukwatta et al. ${ }^{15}$ and is summarized here. We used 21 3DUS images from 21 subjects for the evaluations. All subjects provided written informed consent to the study protocol, which was approved by the University of Western Ontario Research Ethics Board. To better represent a diverse subject group, images were blindly selected from three subject groups; seven images from diabetes subjects, seven images from rheumatoid arthritis subjects, and seven images from atorvastatin subjects who have carotid stenosis of $60 \%$ or more. ${ }^{15}$ The presence of stenosis was confirmed using carotid Doppler US flow velocities.

The mechanical 3DUS acquisition system developed in our laboratory has been described previously ${ }^{7,42}$ and is summarized here. The 3DUS images (voxel size $\approx 0.1 \times 0.1$ $\times 0.15 \mathrm{~mm}^{3}$ ) were acquired using a L12-5 linear US transducer (Philips, Bothell, WA, USA) with a central frequency of 8.5 MHz attached to an ATL HDI 5000 US machine (Philips, Bothell, WA, USA). The transducer was translated along the patient's neck using a motorized mechanism at a uniform speed of $3 \mathrm{~mm} / \mathrm{s}$ for about $4 \mathrm{~cm}$, without cardiac gating. 3DUS images were reconstructed in real time from the digitized 2D frames acquired at $30 \mathrm{~Hz}$ and displayed using multiplanar reformatting. ${ }^{42}$

\section{RESULTS}

The algorithm was evaluated for accuracy and intraobserver variability using 21 3DUS images with a fixed set of parameters as shown in Table III. All of the results in this section were computed using four anchor points per slice. We report the results in terms of computational time, accuracy, and repeatability as follows.

\section{III.A. Computational time and user interaction}

The reported times were calculated as a mean of the 21 3DUS images, which have been segmented five times. A mean observer time of $8.3 \pm 1.5 \mathrm{~min}$ was required to manually delineate the MAB and LIB from a 3DUS image on 11 transverse $2 \mathrm{D}$ slices. The algorithm required mean observer time of $1.6 \pm 0.3 \mathrm{~min}$ for choosing four anchor points on the MAB and LIB with an ISD of $1 \mathrm{~mm}$ for a 3DUS image with 11 2D slices for segmentation. For the proposed 3D algorithm with an ISD of $1 \mathrm{~mm}$ for initialization, the observer is required to choose 88 points in total to segment the MAB and LIB from a $1 \mathrm{~cm}$ portion of the CCA similar to the $2 \mathrm{D}$ method. ${ }^{15}$ 
However, the number of points required to initialize the algorithm is reduced to $48,32,24$, and 16 points for ISDs of 2, 3, 4 , and $10 \mathrm{~mm}$ for initialization, respectively.

In addition to the observer time for initialization, the algorithm required a mean computational time of $1.14 \pm 0.83 \mathrm{~min}$ to segment a 3DUS image using a nonoptimized MATLAB (Natick, MA, USA) implementation on a PC with Intel core i7 processor (Intel corporation, Santa Clara, CA) with a speed of $3.0 \mathrm{GHz}$. From the total computational time, mean computational time of $0.91 \pm 0.8 \mathrm{~min}$ is used for computing local region-based image force for the MAB segmentation.

\section{III.B. Accuracy}

The slice-by-slice comparison of algorithm to manual segmentations for two 3DUS images is shown in Figs. 6 and 7, one with mild stenosis and one with moderate stenosis. Each column corresponds to the algorithm segmentation results generated with the corresponding ISD. The mean manual segmentations are shown as yellow continuous contours and the mean algorithm segmentations are shown as purple dashed contours. A single round of algorithm segmentations, along with the anchor points used for initialization, is shown as cyan dashed contours. For example, for an ISD of $2 \mathrm{~mm}$, every other slice is initialized, whereas for an ISD of $10 \mathrm{~mm}$ only the first and the last slices are initialized. For the 3DUS image with a mild stenosis (see Fig. 6), even for an ISD of $10 \mathrm{~mm}$, the algorithm contours for both the MAB and LIB are in good agreement with the manual contours. However, for the 3DUS image with a moderate stenosis (see Fig. 7), the accuracy in terms of DSC for the entire slice set decreased with the increase of ISD, where the lowest DSC was reported for the ISD of $10 \mathrm{~mm}$ for both the MAB and LIB.

The comparison of the algorithm-generated MAB and LIB surfaces with an ISD of $3 \mathrm{~mm}$ to the manually generated surfaces for the same two 3DUS images used in Figs. 6 and 7 are shown in Fig. 8. The algorithm-generated surfaces (shown in purple) are overlaid with the manually generated surfaces (shown in yellow), where the algorithm surfaces appear smoother than the manual segmentations. This is due to the fact that the algorithm incorporated the smoothness energy and generated the MAB and LIB surfaces at a slice thickness

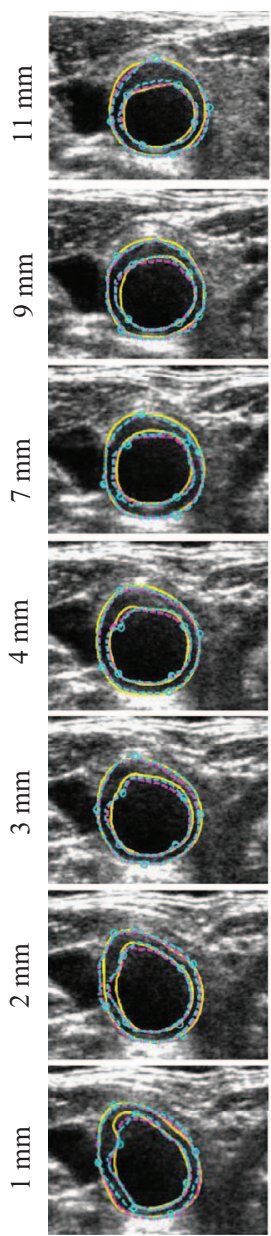

ISD $=1 \mathrm{~mm}$

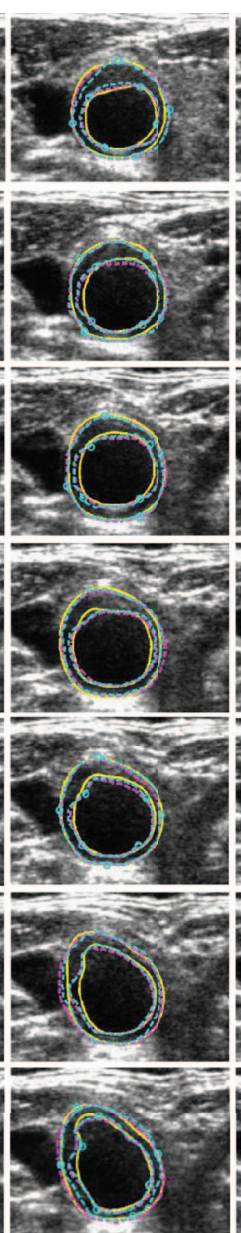

$\mathrm{ISD}=2 \mathrm{~mm}$

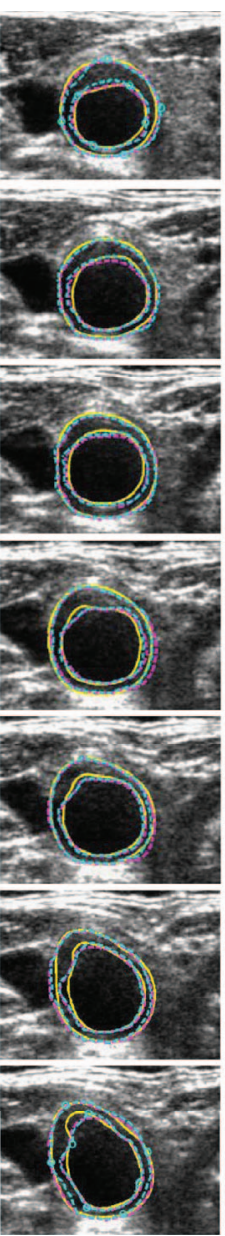

ISD $=3 \mathrm{~mm}$

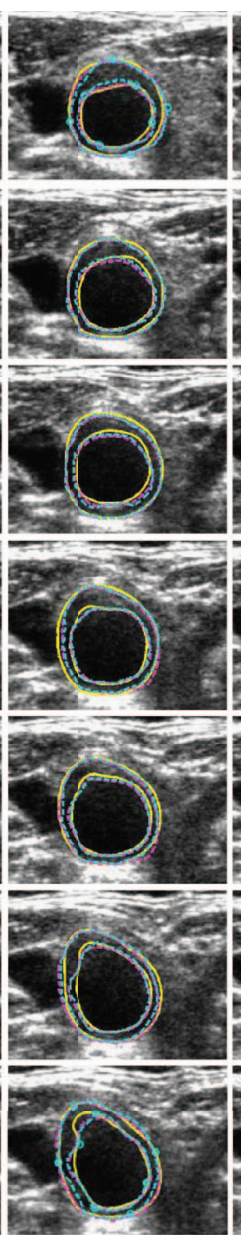

ISD $=4 \mathrm{~mm}$

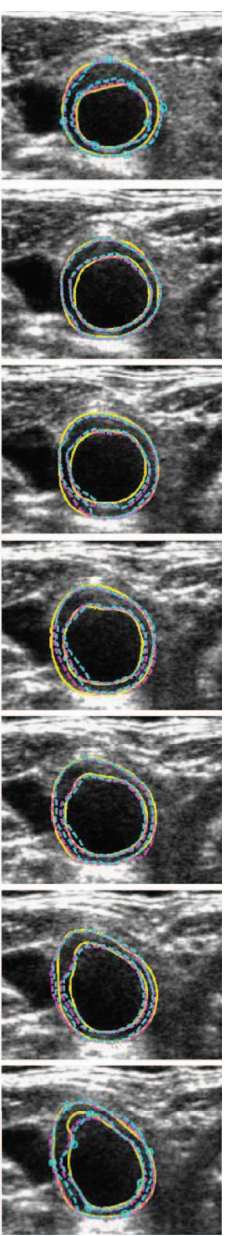

$\mathrm{ISD}=10 \mathrm{~mm}$

FIG. 6. 2D slice-by-slice comparisons of algorithm segmentations to manual segmentations for a subject with a mild stenosis. Results for ISD from 1 to $4 \mathrm{~mm}$, and $10 \mathrm{~mm}$ are shown. The contours are as follows: Continuous yellow contour--mean manual MAB and LIB, dashed purple contour--mean algorithm MAB and LIB, and cyan dashed contour--one round of algorithm MAB and LIB. Each row corresponds to the distance from the bifurcation (BF) and each column corresponds to the ISD used for initialization. 


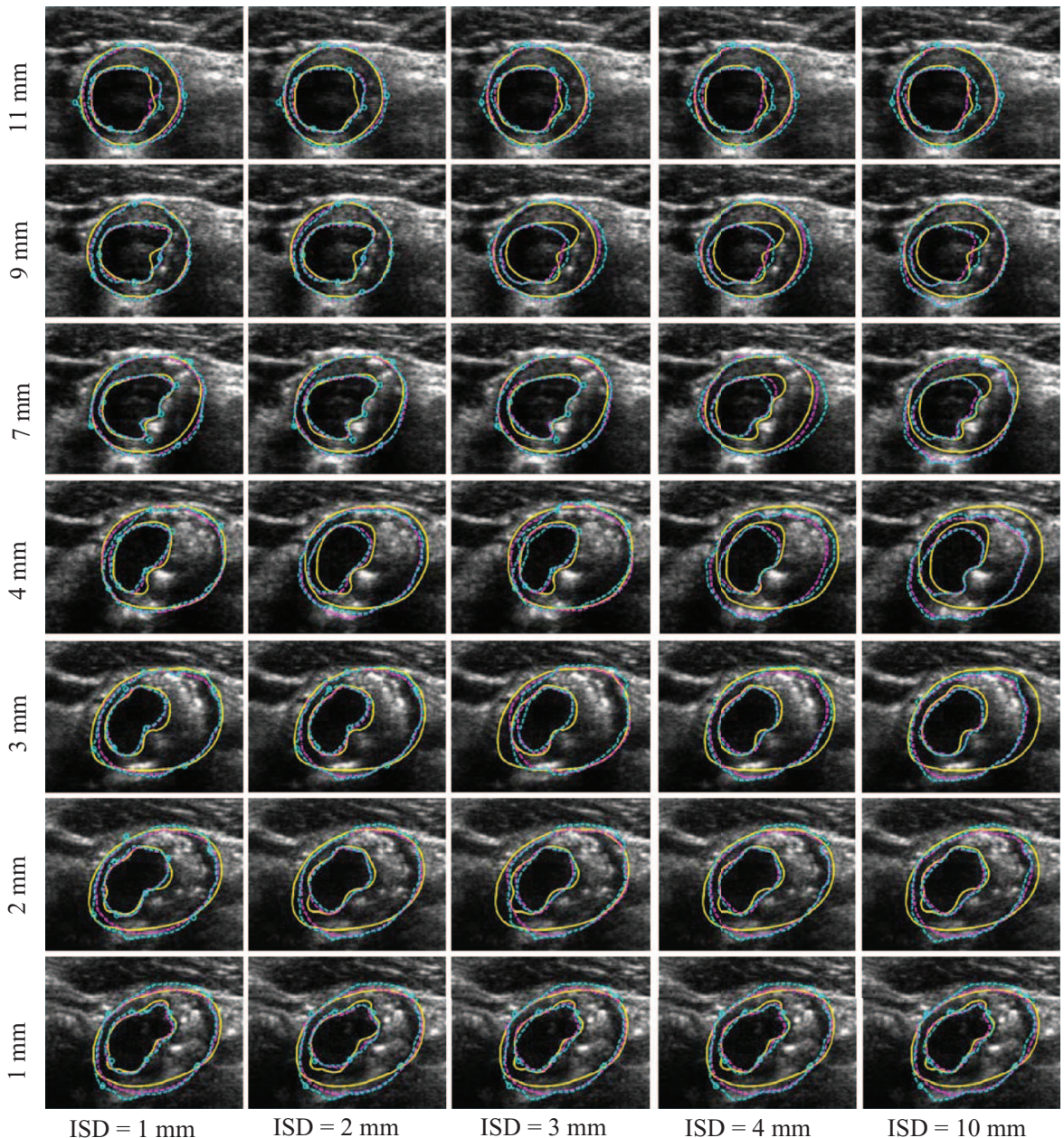

FIG. 7. 2D slice-by-slice comparisons of algorithm segmentations to manual segmentations for a subject with a moderate stenosis (stenosis is between $30 \%$ and 70\%). Results for ISD from 1 to $4 \mathrm{~mm}$ and $10 \mathrm{~mm}$ are shown. The accuracy dropped at 4 and $10 \mathrm{~mm}$. The contours are as follows: Continuous yellow contour--mean manual MAB and LIB, dashed purple contour-mean algorithm MAB and LIB, and cyan dashed contour--one round of algorithm MAB and LIB. Each row corresponds to the distance from the BF and each column corresponds to the ISD used for initialization.

of $0.15 \mathrm{~mm}$, while the manual segmentations were generated at an ISD of $1 \mathrm{~mm}$.

The corresponding flattened VWT maps ${ }^{45}$ for the algorithm and the manual segmentations for the same two 3DUS images are shown in Fig. 9. In the flattened VWT map, the color encodes the thickness between the MAB and LIB. Visually, the algorithm-generated flattened VWT map closely resembles the manual flattened VWT map.

The region-based and distance-based metrics results for the MAB and LIB for the 21 3DUS images are shown in Table IV. The results of the 2D segmentation method ${ }^{15}$ are also included in Table IV for a direct comparison with the proposed 3D algorithm. We evaluated the accuracy of the algorithm with varying ISDs from 1 to 4 and $10 \mathrm{~mm}$. The metrics were computed by comparing the mean algorithm boundary with the mean manual boundary obtained from five repeated segmentations. The highest mean DSC for the MAB and LIB for the proposed 3D algorithm was reported for the ISD of $1 \mathrm{~mm}$. As expected, the DSC gradually decreased with the increasing ISD for both the carotid MAB and LIB. At all ISDs, the MAB yielded a higher DSC than the LIB. The standard deviations (SD) of DSC were small and gradually increased with the ISD for both the MAB and LIB, except at ISD of $4 \mathrm{~mm}$ for the LIB. Paired $t$-tests have been performed to evaluate the statistical significance of the DSC measurements of the $2 \mathrm{D}$ method $^{15}$ and the proposed $3 \mathrm{D}$ method. The 3D algorithm is statistically significantly different from the $2 \mathrm{D}$ method at all the ISDs tested.

Similar to the trend in DSC results, both MAD and MAXD errors increased with increasing ISD for both the MAB and LIB. Similar to the 2D method, ${ }^{15}$ the 3D algorithm yielded submillimeter errors for MAD and its SDs for all ISDs. Except for MAD at ISD of $1 \mathrm{~mm}$, the MAD errors of the 3D algorithm were statistically significantly different from the MAD errors of the 2D method. ${ }^{15}$ The MAXD errors ranged from 0.75 to $1.19 \mathrm{~mm}$ for the MAB and 0.3 to $1.49 \mathrm{~mm}$ for the LIB, where the highest MAXD was reported for ISD of $10 \mathrm{~mm}$. Except for the MAXD errors for the MAB at the ISD of $1 \mathrm{~mm}$, the MAXD errors of the 3D algorithm were significantly different from the MAXD errors of the 2D method. ${ }^{15}$

Table V shows the volume-based evaluation results for the 3D algorithm for ISDs from 1 to 4 and $10 \mathrm{~mm}$. The results of the $2 \mathrm{D}$ segmentation method ${ }^{15}$ are also included in Table V for direct comparison. For the VWV measurements, 


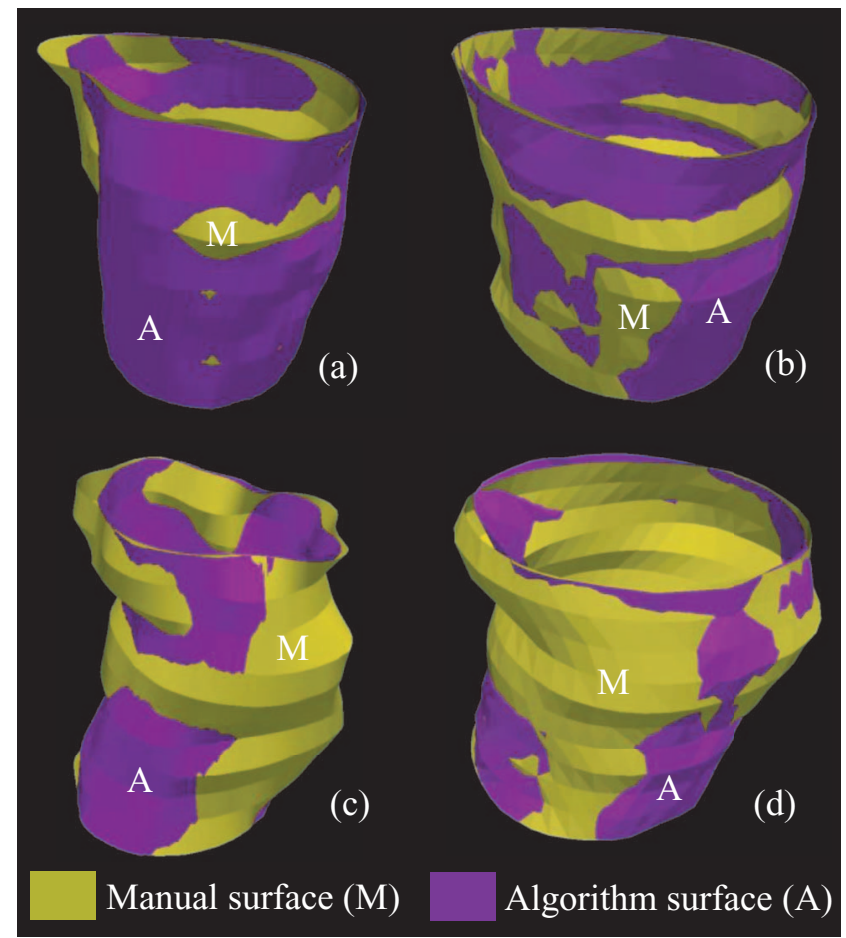

FIG. 8. Comparison of the MAB and LIB algorithm segmentations to manual segmentations for an ISD of $3 \mathrm{~mm}$ for two example 3DUS images that were used for Figs. 6 and 7. The algorithm-generated surfaces are denoted by the letter ' $A$ ' and manually generated surfaces are denoted by the letter ' $M$ '. (a) LIB surface comparison with manual segmentation for a subject with a mild stenosis. (b) MAB surface comparison with manual segmentation of the same subject. (c) LIB surface comparison with manual segmentation for a subject with a moderate stenosis. (d) MAB surface comparison with manual segmentation of the same subject.

the algorithm underestimated the volumes for ISD of 1, 4, and $10 \mathrm{~mm}$ and slightly overestimated for ISD of 2 and $3 \mathrm{~mm}$. Similar to the trend in the region-based and distance-based evaluation results, the absolute VWV error $\left|\delta V_{\mathrm{VWV}}\right|$ gradually increased from $4.32 \%$ to $13.28 \%$ with the increase of ISD. The absolute volumes errors ( $\left|\delta V_{\mathrm{VWV}}\right|,\left|\delta V_{\mathrm{MAB}}\right|$, and $\left.\left|\delta V_{\mathrm{LIB}}\right|\right)$ for the proposed $3 \mathrm{D}$ algorithm were not statistically significantly different from the results of the 2D segmentation for the ISDs of 1 to $3 \mathrm{~mm}$. However, the $\left|\delta V_{\mathrm{VwV}}\right|$ results for the ISDs of 4 and $10 \mathrm{~mm}$ were significantly different.
The $p$-values for the hypothesis test that compares the mean VWV of the algorithm and manual segmentation using a two-way repeated measures ANOVA are shown in Table VI, along with the mean difference and the confidence interval (CI) of the mean difference of the algorithm- and manually generated VWV. As indicated by the $p$-values in Table VI, the algorithm failed to show a statistically significant difference for ISDs of $1,2,3$, and $4 \mathrm{~mm}$, with respect to the manually generated VWV. However, manually and algorithm-generated VWVs at ISD of $10 \mathrm{~mm}$ are significantly different. In comparison to the $2 \mathrm{D}$ method, ${ }^{15}$ the $3 \mathrm{D}$ algorithm also failed to show a significant difference for ISDs of 1,2 , and $3 \mathrm{~mm}$, while there was significant difference for ISDs of 4 and $10 \mathrm{~mm}$. Figure 10 shows the Bland-Altman plot $^{46}$ for the comparison of algorithm- and manually generated VWV for an ISD of $3 \mathrm{~mm}$. The algorithm has a bias of $0.64 \mathrm{~mm}^{3}$ and a CI of -23.5 to $22.7 \mathrm{~mm}^{3}$, which is relatively small compared to the 244-926 $\mathrm{mm}^{3}$ range of the VWV. An example correlation plot for algorithm- and manually generated VWV is shown for ISD of $3 \mathrm{~mm}$ in Fig. 11. The black dashed line indicates the $95 \%$ CI of Pearson correlation coefficient ( $\mathrm{r}$ ). The Pearson correlation coefficient is also shown in Table VI, along with their $p$-values and 95\% CI. The correlation coefficient of algorithm- and manually generated VWVs were in the range of 0.95 to 0.97 for ISDs of 1 to $4 \mathrm{~mm}$ and 0.89 for ISD of $10 \mathrm{~mm}$. They were also significantly correlated ( $p<0.0001)$ with manually generated VWVs for all tested ISDs.

\section{III.C. Reproducibility}

The intraobserver variability results of generating MAB, LIB volumes, and VWV are shown in Table VII. The results of the previous 2D method ${ }^{15}$ are also shown in Table VII for direct comparison. The manual segmentation yielded the smallest $\mathrm{CV}_{\mathrm{VWV}}$ and $\mathrm{CV}_{\mathrm{MAB}}$, although the proposed $3 \mathrm{D}$ algorithm reported the smallest $\mathrm{CV}_{\mathrm{LIB}}$ at $2 \mathrm{~mm}$. The $\mathrm{CV}_{\mathrm{VWV}}$, $\mathrm{CV}_{\mathrm{MAB}}$, and MDD gradually increased with the increase in ISD for the proposed algorithm. The $\mathrm{CV}_{\mathrm{LIB}}$ has a similar trend, except at $2 \mathrm{~mm}$. The CVs for the 3D algorithm, especially for ISDs of 1 and $2 \mathrm{~mm}$, are comparable to the CVs of the 2D algorithm. The MDDs of the proposed 3D algorithm were in the range of 57.8 to $112.9 \mathrm{~mm}^{3}$, and the MDD

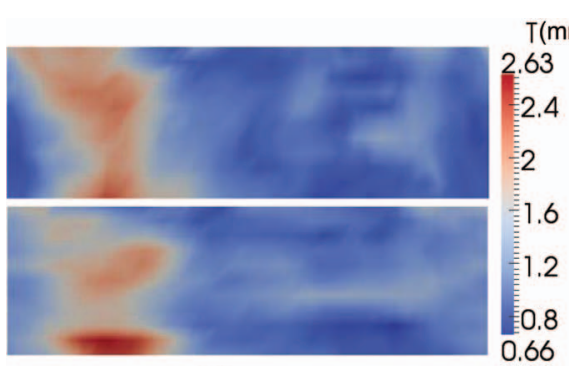

(a)

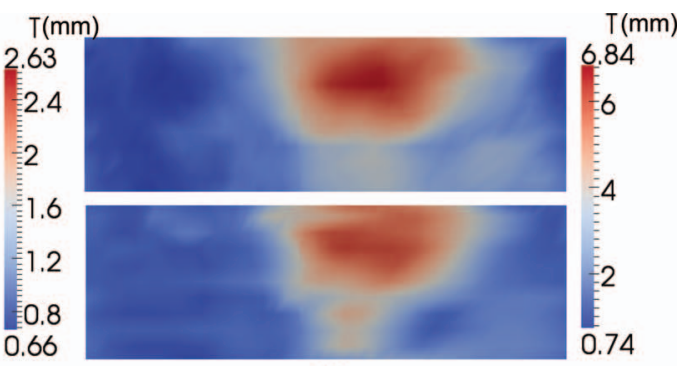

(b)

FIG. 9. Algorithm and manually generated flattened VWT maps of the surfaces shown in Fig. 8 for the same two example 3DUS images. The first row corresponds to algorithm-generated flattened VWT maps, whereas second row corresponds to manually generated flattened VWT maps. (a) Subject with a mild stenosis. (b) Subject with a moderate stenosis. 
TABLE IV. Results for the MAB and LIB segmentation using region-based and distance-based metrics for the 21 3DUS images using the average boundaries. The results of the 3D algorithm are given for ISD of 1, 2, 3, 4, and $10 \mathrm{~mm}$.

\begin{tabular}{|c|c|c|c|c|c|c|}
\hline \multirow[b]{2}{*}{ Metric } & \multirow[b]{2}{*}{ 2D method (Ref. 15) } & \multicolumn{5}{|c|}{ 3D method (ISD varied from 1 to 4 , and $10 \mathrm{~mm}$ ) } \\
\hline & & $1 \mathrm{~mm}$ & $2 \mathrm{~mm}$ & $3 \mathrm{~mm}$ & $4 \mathrm{~mm}$ & $10 \mathrm{~mm}$ \\
\hline $\operatorname{DSC}_{\mathrm{MAB}}(\%)$ & $95.4 \pm 1.6$ & $95.03 \pm 1.77$ & $94.57 \pm 2.01$ & $94.4 \pm 2.24$ & $93.53 \pm 3.83$ & $90.4 \pm 4.3$ \\
\hline $\mathrm{DSC}_{\mathrm{LIB}}(\%)$ & $93.1 \pm 3.1$ & $92.09 \pm 4.23$ & $91.08 \pm 4.46$ & $90.64 \pm 4.97$ & $89.24 \pm 5.87$ & $84.85 \pm 5.6$ \\
\hline $\operatorname{MAD}_{\mathrm{MAB}}(\mathrm{mm})$ & $0.2 \pm 0.1$ & $0.24 \pm 0.08$ & $0.27 \pm 0.10$ & $0.28 \pm 0.18$ & $0.31 \pm 0.19$ & $0.46 \pm 0.23$ \\
\hline $\operatorname{MAD}_{\text {LIB }}(\mathrm{mm})$ & $0.2 \pm 0.1$ & $0.22 \pm 0.10$ & $0.34 \pm 0.16$ & $0.35 \pm 0.16$ & $0.40 \pm 0.19$ & $0.54 \pm 0.26$ \\
\hline $\operatorname{MAXD}_{\mathrm{MAB}}(\mathrm{mm})$ & $0.6 \pm 0.3$ & $0.75 \pm 0.69$ & $0.83 \pm 0.85$ & $0.89 \pm 0.82$ & $0.92 \pm 1.00$ & $1.19 \pm 0.87$ \\
\hline MAXD $_{\text {LIB }}(\mathrm{mm})$ & $0.7 \pm 0.6$ & $0.3 \pm 1.05$ & $0.91 \pm 0.51$ & $0.97 \pm 0.62$ & $1.13 \pm 0.82$ & $1.49 \pm 0.97$ \\
\hline
\end{tabular}

TABLE V. Results for the VWV, MAB, and LIB using volume-based metrics for the 21 3DUS images. The results of the 3D algorithm are given for ISD of 1, $2,3,4$, and $10 \mathrm{~mm}$

3D method (ISD from 1 to 4 , and $10 \mathrm{~mm}$ )

\begin{tabular}{|c|c|c|c|c|c|c|}
\hline Metric & 2D method (Ref. 15) & $1 \mathrm{~mm}$ & $2 \mathrm{~mm}$ & $3 \mathrm{~mm}$ & $4 \mathrm{~mm}$ & $10 \mathrm{~mm}$ \\
\hline$\delta \mathrm{V}_{\mathrm{VWV}}(\%)$ & $-0.9 \pm 6.6$ & $-3.22 \pm 9.65$ & $1.02 \pm 12.32$ & $0.56 \pm 12.42$ & $-5.5 \pm 13.1$ & $-12.7 \pm 17.65$ \\
\hline$\delta \mathrm{V}_{\mathrm{VWV}}\left(\mathrm{mm}^{3}\right)$ & $-5.08 \pm 26.5$ & $-15.45 \pm 38.9$ & $2.28 \pm 47.44$ & $0.64 \pm 49.54$ & $-23.60 \pm 52.47$ & $-59.8 \pm 73.5$ \\
\hline$\delta \mathrm{V}_{\mathrm{MAB}}(\%)$ & $-1.3 \pm 2.8$ & $-0.03 \pm 3.08$ & $2.28 \pm 3.1$ & $0.85 \pm 9.25$ & $-2.97 \pm 4.12$ & $-9.91 \pm 7.11$ \\
\hline$\delta \mathrm{V}_{\text {LIB }}(\%)$ & $-1.0 \pm 6.4$ & $3.60 \pm 8.60$ & $3.10 \pm 4.0$ & $-0.37 \pm 49.5$ & $-0.75 \pm 12.42$ & $-6.65 \pm 17.16$ \\
\hline$\left|\delta \mathrm{V}_{\mathrm{VwV}}\right|(\%)$ & $5.0 \pm 4.3$ & $4.32 \pm 7.20$ & $5.6 \pm 8.5$ & $5.64 \pm 8.1$ & $6.29 \pm 9.78$ & $13.28 \pm 12.30$ \\
\hline$\left|\delta \mathrm{V}_{\mathrm{MAB}}\right|(\%)$ & $2.5 \pm 1.8$ & $1.92 \pm 1.69$ & $2.53 \pm 2.17$ & $1.55 \pm 2.45$ & $2.79 \pm 2.93$ & $8.12 \pm 6.50$ \\
\hline$\left|\delta \mathrm{V}_{\mathrm{LIB}}\right|(\%)$ & $5.6 \pm 3.0$ & $2.8 \pm 7.18$ & $3.79 \pm 7.54$ & $4.43 \pm 6.26$ & $6.10 \pm 8.56$ & $10.63 \pm 10.46$ \\
\hline
\end{tabular}

TABLE VI. Comparison of algorithm- and manually generated VWV for 21 3DUS images using statistical testing and Pearson r. The results of the 3D algorithm are given for ISD of $1,2,3,4$, and $10 \mathrm{~mm}$.

\begin{tabular}{|c|c|c|c|c|c|c|}
\hline \multirow[b]{2}{*}{ Metric } & \multirow[b]{2}{*}{ 2D method (Ref. 15) } & \multicolumn{5}{|c|}{ 3D method (ISD from 1 to 4 and $10 \mathrm{~mm}$ ) } \\
\hline & & $1 \mathrm{~mm}$ & $2 \mathrm{~mm}$ & $3 \mathrm{~mm}$ & $4 \mathrm{~mm}$ & $10 \mathrm{~mm}$ \\
\hline$p$-value & 0.41 & 0.09 & 0.82 & 0.97 & 0.058 & 0.002 \\
\hline Mean $\left(\mathrm{mm}^{3}\right)$ & -5.1 & -15.45 & 2.28 & 0.64 & -23.6 & -59.8 \\
\hline $95 \% \mathrm{CI}\left(\mathrm{mm}^{3}\right)$ & $-17.4-7.2$ & $-33.6-2.7$ & $-19.8-24.4$ & $-23.5-22.7$ & $-48.1-0.86$ & $-94.1--25.5$ \\
\hline Pearson $r$ & 0.97 & 0.97 & 0.96 & 0.95 & 0.95 & 0.89 \\
\hline$p$-value & $<0.0001$ & $<0.0001$ & $<0.0001$ & $<0.0001$ & $<0.0001$ & $<0.0001$ \\
\hline $95 \% \mathrm{CI}$ for $\mathrm{r}$ & $0.94-0.99$ & $0.93-0.99$ & $0.91-0.98$ & $0.89-0.98$ & $0.88-0.98$ & $0.75-0.95$ \\
\hline
\end{tabular}

increased with the increasing ISD. The MDD of the 3D algorithm at ISD of $1 \mathrm{~mm}$ is smaller than the 2D method, although the $3 \mathrm{D}$ algorithm reported slightly higher MDDs for ISD of 2 and $3 \mathrm{~mm}$.

\section{DISCUSSION}

In this paper, we developed and evaluated a 3D semiautomated segmentation algorithm based on SFLS method to delineate the MAB and LIB of the CCA for the measurement of the VWV, which is a previously developed 3DUS-based measurement ${ }^{8}$ of carotid atherosclerosis. As such, the purpose of the algorithm is to generate VWV measurements for monitoring patients being managed for risk of stroke using nonsurgical methods. ${ }^{9-11}$ In addition, the proposed algorithm may also be used for generation of VWT change maps ${ }^{12}$ and analysis of vessel wall region for plaque characterization. ${ }^{47,48}$
Here, we discuss the technical details of our 3DUS VWV segmentation algorithm, computational time, measurement accuracy, measurement reproducibility, and comparison to previous methods.

\section{IV.A. Methodology}

The proposed algorithm is an extension of the 2D slicewise segmentation method ${ }^{15}$ that we have described previously. We chose SFLS method over other level set methods for two main reasons. The SFLS method is an improvement to the narrowband level set methods, ${ }^{49}$ which is relatively less computationally expensive than classical level set methods, ${ }^{36,39}$ which compute the level set function for the entire image at each iteration. Moreover, the SFLS method does not split the segmentation into multiple regions away from the zero level, thus disjoint regions are not generated in the segmentation. 


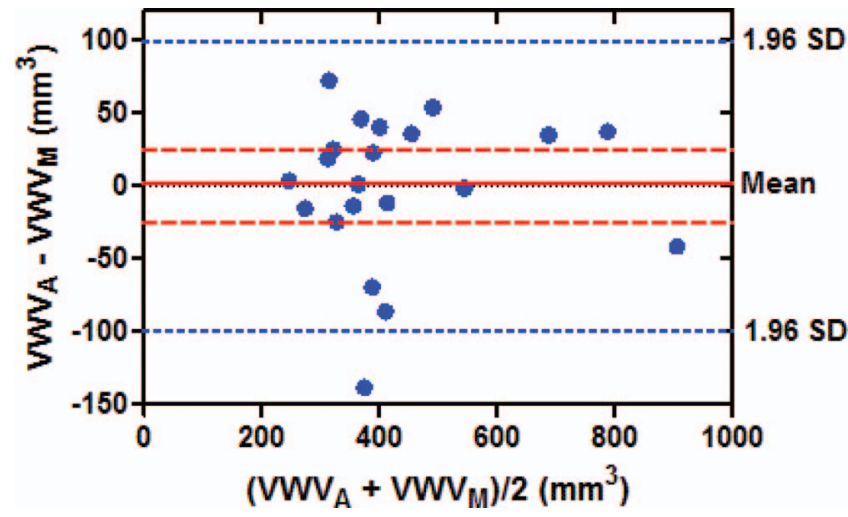

FIG. 10. Bland-Altman plot (Ref. 46) for comparing algorithm- and manually generated 3DUS VWV, where ISD of $3 \mathrm{~mm}$ is used for the algorithm initialization. The red continuous line labeled as mean indicates the bias, the blue dotted lines labeled as $1.96 \mathrm{SD}$ indicate the level of agreement, and the red dashed lines indicate the $95 \%$ CI.

In our paper, we used the minimum and maximum intensity values of the 3DUS image for normalization of the entire image at once, due to both its simplicity and the ability to perform the normalization without any additional user interactions to identify bright intensities around adventitia. However, for a subsequent analysis of the segmented vessel wall region, which is very sensitive to variations in image intensity, such as texture analysis to differentiate plaque components, ${ }^{47}$ a more sophisticated normalization would be required to normalize the images. ${ }^{50,51}$

In the experiments, the observer chose four anchor points on initialization boundaries for any chosen ISD. However, in practice, the observer can choose more anchor points on each initialization boundary. The observer can also vary the ISD for each 3DUS image depending on the amount of plaque and the complexity of the plaque surface. We speculate that the accuracy and reproducibility of the segmentation may increase with the increasing number of anchor points, as indicated by Fig. 9 in the 2D segmentation paper. ${ }^{15}$

The 3D algorithm has been validated on 3DUS images from a single ultrasound device. However, different scanners could generate 3DUS images with different voxel sizes. The differences in voxel size across images do not affect the segmentation, because the thresholds and parameters of the algorithm are defined in millimeters. Such millimeter values are converted to voxel values of the current 3DUS image during the segmentation procedure.

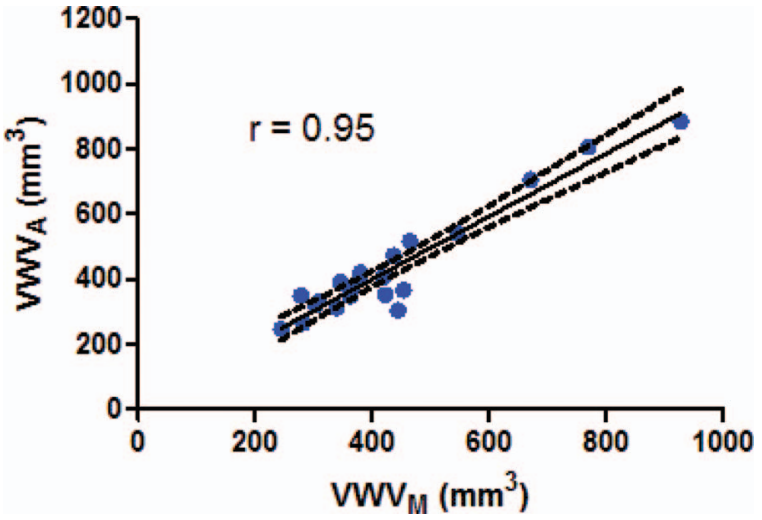

FIG. 11. Correlation plot for algorithm- and manually generated 3DUS VWV, where ISD of $3 \mathrm{~mm}$ is used for the algorithm initialization. The dashed lines indicate the $95 \% \mathrm{CI}$ of the best fit line.

The proposed 3D algorithm has some limitations. Although, the $3 \mathrm{D}$ algorithm reduces user interactions required for segmentation with an ISD greater than $1 \mathrm{~mm}$ in contrast to the $2 \mathrm{D}$ method, ${ }^{15}$ the $3 \mathrm{D}$ algorithm is still far from full automation due to the challenges in the segmentation of very low-contrast boundaries. In addition, the initial surface for the $\mathrm{MAB}$ is required to be close to the actual MAB for obtaining accurate segmentations, because the effect of the local regionbased term is limited by its local radius $r_{L}$.

\section{IV.B. Computational time}

The algorithm required lesser user time and fewer interactions to generate segmentations than manual delineations. The algorithm required a mean time of $1.6 \mathrm{~min}$ to initialize the MAB and LIB of the CCA from a 3DUS image for a portion of $1 \mathrm{~cm}$ with an ISD of $1 \mathrm{~mm}$. In our experiments, the observer provided anchor points with an ISD of $1 \mathrm{~mm}$. For ISDs more than or equal to $2 \mathrm{~mm}$, the anchor points corresponding to the given ISD are chosen from the anchor point set generated with the ISD of $1 \mathrm{~mm}$. If we assume that number of points used for initialization is linearly proportional to the time taken for the observer to actually choose anchor points, an observer would take approximately $0.58 \mathrm{~min}$ to initialize a $1 \mathrm{~cm}$ portion of the CCA with an ISD of $3 \mathrm{~mm}$. In this case, the algorithm would require only $1.72 \mathrm{~min}$ of total time, which comprises of user interaction time of $0.58 \mathrm{~min}$ and SFLS algorithm computational time of $1.14 \mathrm{~min}$. This is approximately $79 \%$

TABLE VII. Standard deviation (SD), coefficient of variation (CV), and minimum detectable difference (MDD) of volume measurements for 21 3DUS images computed using the repeated measurements of algorithm and manual segmentations. The results of the 3D algorithm are given for ISD of $1,2,3$, 4 , and 10 mm.

\begin{tabular}{|c|c|c|c|c|c|c|c|}
\hline \multirow[b]{2}{*}{ Metric } & \multirow[b]{2}{*}{ Manual } & \multirow[b]{2}{*}{ 2D method (Ref. 15) } & \multicolumn{5}{|c|}{ 3D method (ISD from 1 to 4 and $10 \mathrm{~mm}$ ) } \\
\hline & & & $1 \mathrm{~mm}$ & $2 \mathrm{~mm}$ & $3 \mathrm{~mm}$ & $4 \mathrm{~mm}$ & $10 \mathrm{~mm}$ \\
\hline $\operatorname{SD}_{V W V}\left(\mathrm{~mm}^{3}\right)$ & 18.1 & 23.2 & 20.85 & 24.35 & 27.13 & 34.0 & 40.7 \\
\hline $\mathrm{CV}_{\text {VwV }}(\%)$ & 3.89 & 5.1 & 4.97 & 5.57 & 6.8 & 8.23 & 10.82 \\
\hline $\mathrm{CV}_{\text {MAB }}(\%)$ & 1.53 & 2.36 & 2.47 & 2.78 & 3.4 & 3.6 & 5.25 \\
\hline $\mathrm{CV}_{\mathrm{LIB}}(\%)$ & 3.30 & 3.5 & 3.12 & 2.34 & 3.7 & 4.88 & 6.18 \\
\hline $\operatorname{MDD}_{\mathrm{VWV}}\left(\mathrm{mm}^{3}\right)$ & 50.3 & 64.2 & 57.8 & 67.5 & 75.2 & 94.2 & 112.9 \\
\hline
\end{tabular}


reduction in time (1.72 vs $8.3 \mathrm{~min})$ in comparison to manual segmentation.

In comparison to the previous $2 \mathrm{D}$ method, ${ }^{15}$ the $3 \mathrm{D}$ algorithm was able to reduce user interaction further, when ISD of more than or equal to $2 \mathrm{~mm}$ is used for the initialization. For example, with an ISD of $3 \mathrm{~mm}$, the observer is required to initialize only 32 points in total on four slices for both the MAB and LIB as opposed to 88 points in 11 slices for segmenting the MAB and LIB from a $1 \mathrm{~cm}$ section of the CCA from a given 3DUS image. However, with the current nonoptimized MATLAB implementation of the SFLS method, the proposed 3D algorithm required approximately similar computational time to the 2D method ${ }^{15}(1.14 \pm 0.83$ vs $1.2 \pm 0.2 \mathrm{~min}$ ). The main time-consuming task for the $3 \mathrm{D}$ algorithm is the local region-based force computation for the MAB segmentation, which requires about $80 \%$ ( 0.92 out of $1.14 \mathrm{~min}$ ) of the computational time. However, the local region-based force calculation can be performed independently for each point on the zero level set, thus the computation can be parallelized in a graphics processing unit (GPU), which may be able to substantially speed up the segmentation.

\section{IV.C. Accuracy}

For 3DUS images with mild stenosis (see Fig. 6 for an example), accurate segmentations could be obtained even at ISD of $10 \mathrm{~mm}$, because the CCA is more regular from one slice to the next over a $1 \mathrm{~cm}$ distance of the CCA. However, when the subject has moderate (see Fig. 7) or severe stenosis, the morphology of the artery could differ from one slice to its adjacent slice. Therefore, when an ISD, such as $10 \mathrm{~mm}$ is used, the generated initial surface for the 3D segmentation could be too far from the desired boundary for the algorithm to converge to the desired boundary.

The accuracy of the algorithm was evaluated with respect to distance-based, region-based, and volume-based metrics. The algorithm yielded DSCs in the range of $90.4-95.0 \mathrm{~mm}^{3}$ for the MAB and $84.8-92.1 \mathrm{~mm}^{3}$ for the LIB, where the DSC was highest for the ISD of $1 \mathrm{~mm}$, for both the MAB and LIB, and then gradually decreased with the increase of ISD (see Table IV). Moreover, the 3D algorithm is statistically significantly different from the 2D method ${ }^{15}$ at all the ISDs tested.

Similar to the trend in DSC results, both the MAD and MAXD errors were small and increased with increasing ISD for both the MAB and LIB (see Table IV). This may be due to the fact that the initial surface could be further away from desired segmentation with an increasing ISD, thus the algorithm could be trapped in local minima providing suboptimal results. To investigate the reduction in accuracy with increasing ISD, we computed the MAD error between the initial contours and the manual segmentation. The results show an increase in the MAD error with the ISD, with the highest being at an ISD of $10 \mathrm{~mm}$. Except for the MAD at ISD of $1 \mathrm{~mm}$, the MAD errors of the 3D algorithm were statistically significantly different from the MAD errors of 2D method. ${ }^{15}$ The MAXD errors of the 3D algorithm were statistically significantly different from the MAXD errors of 2D method, ${ }^{15}$ except for the MAB at the ISD of $1 \mathrm{~mm}$. The MAB always yielded a higher DSC than the LIB, because the observer may be able to locate anchor points for the MAB more robustly than for the LIB due to its regular shape. This is further supported by the fact that manual $\mathrm{CV}_{\mathrm{MAB}}$ was smaller than manual $\mathrm{CV}_{\mathrm{LIB}}(1.53 \%$ vs $3.30 \%$ as shown in Table VII).

The main purpose of the 3D algorithm is for generating VWV measurements for monitoring plaque burden. Therefore, we extensively validated the accuracy and reproducibility of the algorithm for generating the VWV. The algorithmgenerated VWV was significantly correlated $(p<0.0001)$ with the manually generated VWV $(r>0.95$ for ISDs of 1 to $4 \mathrm{~mm}$, and $\mathrm{r}=0.89$ for ISD of $10 \mathrm{~mm}$ ) with small $95 \%$ $\mathrm{CI}$ for Pearson $\mathrm{r}$ (see Table VI). We failed to detect a statistically significant difference $(p>0.05)$ between algorithmand manually generated VWV for ISD of 1 to $4 \mathrm{~mm}$, although they were statistically significant for ISD of $10 \mathrm{~mm}$. The values within the $95 \%$ CIs (i.e., CI of $-23.5-22.7 \mathrm{~mm}^{3}$ for ISD of $3 \mathrm{~mm}$ ) of the VWV difference were small for ISDs of 1 to $4 \mathrm{~mm}$, in comparison to their VWV range of $244-926 \mathrm{~mm}^{3}$.

We computed both absolute and signed volume errors (see Table V) as volume-based metrics to evaluate the algorithm. The signed volume error provides an indication of the bias of the algorithm, whereas the absolute volume error is a more stringent measure of the discrepancy between algorithm and manual measurements. The absolute VWV errors $\left(\left|\delta V_{\mathrm{VwV}}\right|\right)$ of the proposed $3 \mathrm{D}$ algorithm were in the range of $4.32 \%-$ $13.28 \%$. However, $\left|\delta V_{\mathrm{VwV}}\right|$ of the $3 \mathrm{D}$ algorithm were not significantly different from $\left|\delta V_{\mathrm{VWV}}\right|$ of the $2 \mathrm{D}$ method for the ISDs of $1-3 \mathrm{~mm}$. However, the $\left|\delta V_{\mathrm{VwV}}\right|$ results for the ISDs of 4 and $10 \mathrm{~mm}$ were significantly different. For the ISD of 1,4 , and $10 \mathrm{~mm}$, the algorithm underestimated the VWV. The underestimation of VWV could be mainly due to either the MAB volume is underestimated and/or the LIB volume is overestimated, except for ISD of $10 \mathrm{~mm}$, where both the MAB and LIB volumes were underestimated (see Table V). For ISD of $1 \mathrm{~mm}$, the underestimation of VWV occurred mainly due to the overestimation of the LIB. For ISD of 4 and $10 \mathrm{~mm}$, the underestimation occurred due to the underestimation of the MAB (see Table V). We observed that for ISDs more than $2 \mathrm{~mm}$, the initial surface is mostly enclosed within the desired surface. When the initial surface is further away from the desired boundary, the MAB segmentation could be trapped in local minima because its region-based energy is dependent only on local statistics, which finally leads to underestimating the MAB volume. Although global-region based energy is used for LIB segmentation to avoid this issue, it is not used for the MAB segmentation, because of inhomogeneity of the inside and outside intensities for the MAB. However, since LIB is constrained by the MAB segmentation, when the MAB volume is underestimated, the LIB volume may also be underestimated.

\section{IV.D. Reproducibility}

We used CV and the MDD (see Table VII) to evaluate the intraobserver variability of the algorithm for computing VWV from repeated measurements. The algorithm yielded small 
$\mathrm{CV}_{\mathrm{VWV}}$ (e.g., $5.57 \%$ for ISD of $3 \mathrm{~mm}$ ) that are comparable with the CVs for manual segmentation and the 2D method. ${ }^{15}$ The $\mathrm{CV}_{\mathrm{VWV}}$ also increased with the increase of ISD, which may be due to larger differences in initial surfaces between repetitions for large ISD than for small ISD. Two sample Ftests were carried out to determine the statistical significant difference of the variance of the proposed method with manual segmentation and the $2 \mathrm{D}$ method. ${ }^{15}$ The F-tests failed to show that the variances of the proposed methods are significantly different from the variances of the manual segmentation and the 2D method ${ }^{15}$ for all the ISDs.

Manual $\mathrm{CV}_{\mathrm{VwV}}(3.89 \%)$ was comparable with the previously reported manual intraobserver $\mathrm{CV}_{\mathrm{VwV}}$ values of $4.6 \%$ by Egger et al. ${ }^{8}$ and $4.7 \%$ by Egger et al. ${ }^{52}$ Apart from the effect caused by different datasets, the reason for slightly lower manual $\mathrm{CV}_{\mathrm{VwV}}$ of ours could be due to two main reasons. In our study, the observer sets the BF and axis for each 3DUS image only once and used them for other repetitions, as opposed to setting different $\mathrm{BF}$ and axis for each repetition. In addition, our study used only VWV of the CCA, whereas their study used VWV of internal carotid (ICA) and external carotid arteries (ECA) in addition to VWV of CCA.

Manual $\mathrm{CV}_{\mathrm{VwV}}$ was also smaller than the algorithm $\mathrm{CV}_{\text {VWV }}(3.9 \%$ vs $4.97 \%-10.82 \%)$. Although, manual $\mathrm{CV}_{\mathrm{MAB}}$ was smaller than algorithm $\mathrm{CV}_{\mathrm{MAB}}$, manual $\mathrm{CV}_{\mathrm{LIB}}$ is larger than the algorithm $\mathrm{CV}_{\mathrm{LIB}}$ for ISD of 1 and $2 \mathrm{~mm}$. In addition, $\mathrm{CV}_{\mathrm{MAB}}$ was smaller than the corresponding $\mathrm{CV}_{\mathrm{LIB}}$ in most cases except for the ISD of $2 \mathrm{~mm}$ (see Table VII). This could be due to the fact that the observer can more accurately locate the MAB for choosing anchor points, because of its smooth and low order shape. The MDD values (see Table VII) of the proposed algorithm increased with increased ISD. Although algorithm MDDs were larger than the manual MDD, the MDD results of the proposed algorithm was smaller than a previously reported VWV change of $\approx 120 \mathrm{~mm}^{3} / \mathrm{yr},{ }^{10}$ which suggests that a follow up period of $1 \mathrm{yr}$ could be used for any ISD, although, for ISD of 1 and $2 \mathrm{~mm}$, even 6 month period may be suitable as follow up.

\section{IV.E. Comparison to previous methods}

There are three previous $2 \mathrm{D}$ segmentation methods ${ }^{14-16} \mathrm{re}-$ ported in the literature that segment both the MAB and LIB from 3DUS images as shown in Table I. Out of these two methods, the one proposed by Ukwatta et al. ${ }^{15}$ is currently used in clinical trials. ${ }^{11}$ Therefore, in this paper, we compared the accuracy, intraobserver variability, user interaction, and the computational time of the proposed method to the $2 \mathrm{D}$ segmentation method. ${ }^{15}$ Although the accuracy, intraobserver variability, and computational time of the proposed 3D algorithm is comparable to the $2 \mathrm{D}$ method, ${ }^{15}$ there are two main advantages of the proposed 3D algorithm over previous methods. ${ }^{14,15}$ One advantage is the reduction of number of anchor points for initialization, which translates into overall reduction of total segmentation time. For example, for ISD of $3 \mathrm{~mm}$, the number of anchor points required for segmenting $1 \mathrm{~cm}$ section of the CCA from a 3DUS image is 32, as opposed to 88 with an ISD of $1 \mathrm{~mm}$. The other advantage is the ability to generate the segmentation at each voxel interval (typically $0.15 \mathrm{~mm}$ ) along the long axis direction in a much less time than in a 2D segmentation method. For example, for segmentation of a $1 \mathrm{~cm}$ section of the CCA at ISD of $0.15 \mathrm{~mm}$, the $2 \mathrm{D}$ method $^{15}$ would require 17 min $(67$ slices where 11 slices require $2.8 \mathrm{~min}$ ). However, the proposed algorithm would require about 1.72 min to segment the image at ISD of $0.15 \mathrm{~mm}$ with an initialization ISD of $3 \mathrm{~mm}$.

The 2D segmentation method proposed by Ukwatta et al. ${ }^{15}$ requires a total segmentation time of $2.8 \mathrm{~min}$ and the one proposed by Yang et al. ${ }^{14}$ reported $4.4 \mathrm{~min}$ to segment the CCA from a 3DUS image. The total segmentation time for the proposed algorithm with an ISD of $3 \mathrm{~mm}$ is approximately $1.72 \mathrm{~min}$, which is less than the previous methods. However, note that the algorithm computational times of the proposed 3D method are not directly comparable to the computational times proposed by Yang et al., ${ }^{14}$ due to differences in hardware and datasets used in the papers.

\section{IV.F. Selection of proper ISD}

Based on the evaluation results, more suitable ISDs for initialization are 2 and $3 \mathrm{~mm}$, which use fewer user interactions for initialization than the $2 \mathrm{D}$ method, ${ }^{15}$ while maintaining a DSC of more than $90 \%, \mathrm{CV}_{\mathrm{VwV}}$ less than or equal to $6.8 \%$, $\left|\delta \mathrm{V}_{\mathrm{VWV}}\right|$ less than or equal to $5.64 \%$, and a bias as small as 2.28 and $0.64 \mathrm{~mm}^{3}$, in comparison to VVWs in the range of 244-926 $\mathrm{mm}^{3}$ for VWV computation. After selecting an ISD for a study computing VWV, our results can also be used to estimate the number of subjects that must be monitored in a clinical trial to detect a desired average regression in VWV at a desired power and significance: ${ }^{53,54}$

$$
n \geq \frac{2\left(Z_{\alpha}+Z_{\beta}\right)^{2} \mathrm{SD}_{\mathrm{VWv}}^{2}}{M_{d}^{2}},
$$

where $n$ is the number of subjects, $M_{d}$ is the desired mean difference in VWV, $Z_{\alpha}$ is the standard normal deviate exceeded in either direction with probability $\alpha$, and $Z_{\beta}$ is the standard normal deviate exceeded in one direction with probability $\beta$, where $1-\beta$ is the desired power. ${ }^{53} \mathrm{SD}_{\mathrm{VwV}}$ is the standard deviation of the repeated measurements of VWV as shown in Table VII. For example, for an ISD of $3 \mathrm{~mm}$, $\mathrm{SD}_{\mathrm{VWV}}=27.13 \mathrm{~mm}^{3}, \alpha=0.05$, and $\beta=0.2$ (hence, $Z_{\alpha}$ $=1.96$ and $Z_{\beta}=0.842$ ) to measure a mean VWV regression of $30 \mathrm{~mm}^{3}$ with a $95 \%$ confidence interval, which was in fact the mean VWV regression of a patient group in a statin drug trial $^{10}$ of 3 months, the minimum number of patients required is 13 .

\section{IV.G. Conclusion}

In conclusion, the 3D segmentation algorithm based on SFLS method provided high accuracy (e.g., DSC greater than $90 \%$ for ISDs of 1,2 , and $3 \mathrm{~mm}$ ) in terms of volume-, region-, and distance-based metrics and low intraobserver variability [MDD smaller than previously reported VWV change of $\approx 120 \mathrm{~mm}^{3} / \mathrm{yr}$ (Ref. 10)] in generating the VWV. To our knowledge, this paper is the first attempt to segment both the $\mathrm{MAB}$ and $\mathrm{LIB}$ of the CCA in 3D from 3DUS images for 
the purpose of generating the VWV for monitoring carotid atherosclerosis plaque burden in clinical trials. The method provided comparable accuracy and intraobserver variability results to previous $2 \mathrm{D}$ segmentation method, ${ }^{15}$ with fewer user interactions and shorter segmentation time, when ISD of $2 \mathrm{~mm}$ or more is used for the initialization.

\section{ACKNOWLEDGMENTS}

The authors would like to acknowledge the comments from the anonymous reviews that greatly improved the quality of the paper. The authors thank A. D. Ward for the interesting discussions the authors had about the paper and $\mathrm{S}$. Shavakh for generating the flattened VWT maps. The authors acknowledge the financial support from the Canadian Institutes of Health Research (CIHR) and the Ontario Research Fund (ORF) program. E. Ukwatta acknowledges the support from the Natural Sciences and Engineering Research Council of Canada (NSERC) Canada Graduate Scholarship (CGS). A. Fenster holds a Canada Research Chair in Biomedical Engineering, and acknowledges the support of the Canada Research Chair Program. G. Parraga holds a CIHR New Investigator award and gratefully acknowledges research funding and support from the CIHR.

a) Author to whom correspondence should be addressed. Electronic mail: eukwatta@robarts.ca

${ }^{1}$ V. L. Roger et al., "Heart disease and stroke statistics-2011 update: A report from the American Heart Association," Circulation 123(4), e18-e209 (2011).

2 J. D. Spence and T. Rundek, "Toward clinical applications of carotid ultrasound: Intima-media thickness, plaque area, and three-dimensional phenotypes," in Ultrasound and Carotid Bifurcation Atherosclerosis, edited by A. Nicolaides et al. (Springer, London, 2012), pp. 431-448.

${ }^{3}$ G. Parraga, A. Fenster, A. Krasinski, B. Chiu, M. Egger, and J. D. Spence, "3D carotid ultrasound imaging," in Atheroclerosis Disease Management, edited by J. S. Suri et al. (Springer, New York, 2011), pp. 325-350.

${ }^{4} \mathrm{C}$. Yuan et al., "MRI of atherosclerosis in clinical trials," NMR Biomed. 19(6), 636-654 (2006).

${ }^{5}$ E. Ukwatta, D. Buchanan, G. Parraga, and A. Fenster, "Three-dimensional ultrasound imaging of carotid atherosclerosis," Proceedings of IEEE International conference on Intelligent Computation and Bio-Medical Instrumentation, Wuhan, China, 81-85 (2011).

${ }^{6}$ A. Landry, J. D. Spence, and A. Fenster, "Measurement of carotid plaque volume by 3-dimensional ultrasound," Stroke 35(4), 864-869 (2004).

${ }^{7}$ C. D. Ainsworth, C. C. Blake, A. Tamayo, V. Beletsky, A. Fenster, and J. D. Spence, "3D ultrasound measurement of change in carotid plaque volume: A tool for rapid evaluation of new therapies," Stroke 36(9), 19041909 (2005)

${ }^{8}$ M. Egger, J. D. Spence, A. Fenster, and G. Parraga, "Validation of 3D ultrasound vessel wall volume: An imaging phenotype of carotid atherosclerosis," Ultrasound Med. Biol. 33(6), 905-914 (2007).

${ }^{9}$ I. Shai et al., "Dietary intervention to reverse carotid atherosclerosis," Circulation 121(10), 1200-1208 (2010).

${ }^{10}$ A. Krasinski, B. Chiu, J. D. Spence, A. Fenster, and G. Parraga, "Threedimensional ultrasound quantification of intensive statin treatment of carotid atherosclerosis," Ultrasound Med. Biol. 35(11), 1763-1772 (2009).

${ }^{11}$ D. N. Buchanan, T. Lindenmaier, S. Mckay, Y. Bureau, D. G. Hackam, A. Fenster, and G. Parraga, "The relationship of carotid three-dimensional ultrasound vessel wall volume with age and sex: Comparison to carotid intima-media thickness," Ultrasound Med. Biol. 38(7), 1145-1153 (2012).

${ }^{12}$ B. Chiu, M. Egger, J. D. Spence, G. Parraga, and A. Fenster, "Quantification of carotid vessel wall and plaque thickness change using 3D ultrasound images," Med. Phys. 35(8), 3691-3710 (2008).
${ }^{13}$ C. Mallett, A. A. House, J. D. Spence, A. Fenster, and G. Parraga, "Longitudinal ultrasound evaluation of carotid atherosclerosis in one, two and three dimensions," Ultrasound Med. Biol. 35(3), 367-375 (2009).

${ }^{14}$ X. Yang, J. Jin, W. He, M. Yuchi, and M. Ding, "Segmentation of the common carotid artery with active shape models from 3D ultrasound images," SPIE Medical Imaging: Computer-Aided Diagnosis, San Diego, CA, Vol. 8315, 83152H-1 (2012).

${ }^{15}$ E. Ukwatta et al., "Three-dimensional ultrasound of carotid atherosclerosis: Semi-automated segmentation using a level set-based method," Med. Phys. 38(5), 2479-2493 (2011).

${ }^{16}$ E. Ukwatta, J. Awad, A. D. Ward, D. Buchanan, G. Parraga, and A. Fenster, "Coupled level set approach to segment carotid arteries from 3D ultrasound images," IEEE International Symposium on Biomedical Imaging: From Nano to Macro, Chicago, IL (Institute of Electrical and Electronics Engineers, 2011), pp. 37-40.

${ }^{17}$ F. Molinari, G. Zeng, and J. S. Suri, "A state of the art review on intimamedia thickness (IMT) measurement and wall segmentation techniques for carotid ultrasound," Comput. Methods Programs Biomed. 100(3), 201-221 (2010).

${ }^{18}$ C. P. Loizou, C. S. Pattichis, M. Pantziaris, T. Tyllis, and A. Nicolaides, "Snakes based segmentation of the common carotid artery intima media," Med. Biol. Eng. Comput. 45(1), 35-49 (2007).

${ }^{19}$ F. Molinari, G. Zeng, and J. S. Suri, “An integrated approach to computerbased automated tracing and its validation for 200 common carotid arterial wall ultrasound images: A new technique," J. Ultrasound Med. 29(3), 399-418 (2010).

${ }^{20}$ S. Golemati, J. Stoitsis, E. G. Sifakis, T. Balkizas, and K. S. Nikita, "Using the Hough transform to segment ultrasound images of longitudinal and transverse sections of the carotid artery," Ultrasound Med. Biol. 33(12), 1918-1932 (2007).

${ }^{21}$ F. Mao, J. Gill, D. Downey, and A. Fenster, "Segmentation of carotid artery in ultrasound images: Method development and evaluation technique," Med. Phys. 27(8), 1961-1970 (2000).

${ }^{22}$ A. Zahalka and A. Fenster, "An automated segmentation method for threedimensional carotid ultrasound images," Phys. Med. Biol. 46(4), 13211342 (2001)

${ }^{23}$ P. Abolmaesumi, M. R. Sirouspour, and S. E. Salcudean, "Real-time extraction of carotid artery contours from ultrasound images," in Proceedings of the 13th IEEE Symposium on Computer-Based Medical Systems, Houston, TX (IEEE Computer Society, Washington, DC, 2000), pp. 181-186.

${ }^{24}$ J. Guerrero, S. E. Salcudean, J. A. McEwen, B. S. Masri, and S. Nicolaou, "Real-time vessel segmentation and tracking for ultrasound imaging applications," IEEE Trans. Med. Imaging 26(8), 1079-1090 (2007).

${ }^{25}$ J. D. Gill, H. M. Ladak, D. A. Steinman, and A. Fenster, "Accuracy and variability assessment of a semiautomatic technique for segmentation of the carotid arteries from three-dimensional ultrasound images," Med. Phys. 27(6), 1333-1342 (2000).

${ }^{26}$ I. Solovey, "Segmentation of 3D carotid ultrasound images using weak geometric priors," M.S. thesis, The University of Waterloo, Canada, 2010.

${ }^{27}$ E. Ukwatta, J. Awad, D. Buchanan, G. Parraga, and A. Fenster, "Threedimensional semi-automated segmentation of carotid atherosclerosis from three-dimensional ultrasound images," Proc. SPIE Medical Imaging: Computer-Aided Diagnosis 8315, 831500 (2012).

${ }^{28}$ J. A. Noble and D. Boukerroui, "Ultrasound image segmentation: A survey,” IEEE Trans. Med. Imaging 25(8), 987-1010 (2006).

${ }^{29}$ P.-J. Touboul et al., "Mannheim carotid intima-media thickness consensus (2004-2006)," Cerebrovasc Dis 23(1), 75-80 (2007).

${ }^{30}$ X. Papademetris, A. J. Sinusas, D. P. Dione, and J. S. Duncan, "Estimation of 3D left ventricular deformation from echocardiography," Med. Image Anal. 5(1), 17-28 (2001).

${ }^{31}$ P. Perona and J. Malik, "Scale-space and edge detection using anisotropic diffusion," IEEE Trans. Pattern Anal. 12(7), 629-639 (1990).

${ }^{32}$ Y. Yu and S. T. Acton, "Speckle reducing anisotropic diffusion," IEEE Trans. Image Process. 11(11), 1260-1270 (2002).

${ }^{33}$ J. Canny, "A computational approach to edge detection," IEEE Trans. Pattern Anal. PAMI-8(6), 679-98 (1986).

${ }^{34}$ S. D. Pathak, D. R. Haynor, and Y. Kim, "Edge-guided boundary delineation in prostate ultrasound images," IEEE Trans. Med. Imaging 19(12), 1211-1219 (2000).

${ }^{35}$ R. T. Whitaker, "A level-set approach to $3 \mathrm{D}$ reconstruction from range data,” Int. J. Comput. Vis. 29(3), 203-231 (1998). 
${ }^{36} \mathrm{~S}$. Osher and J. A. Sethian, "Fronts propagating with curvature dependent speed: Algorithms based on Hamilton-Jacobi formulations," J. Comput. Phys. 79(1), 12-49 (1988).

${ }^{37}$ T. Chan and L. Vese, "Active contours without edges," IEEE Trans. Image Process. 10(2), 266-277 (2001).

${ }^{38}$ J. Awad, A. Owrangi, L. Villemaire, E. O'Riordan, G. Parraga, and A. Fenster, "Three-dimensional lung tumor segmentation from x-ray computed tomography using sparse field active models," Med. Phys. 39(2), 851-865 (2012).

${ }^{39} \mathrm{~S}$. Lankton and A. Tannenbaum, "Localizing region-based active contours," IEEE Trans. Image Process. 17(11), 2029-2039 (2008).

${ }^{40}$ V. Caselles, R. Kimmel, and G. Sapiro, "Geodesic active contours," Int. J. Comput. Vis. 22(1), 61-79 (1997).

${ }^{41} \mathrm{~K}$. H. Zou et al., "Statistical validation of image segmentation quality based on a spatial overlap index," Acad. Radiol. 11(2), 178-189 (2004).

${ }^{42}$ A. Fenster, D. B. Downey, and H. N. Cardinal, "Three-dimensional ultrasound imaging," Phys. Med. Biol. 46(5), R67-R99 (2001).

${ }^{43}$ M. Eliasziw, S. L. Young, M. G. Woodbury, and K. Fryday-Field, "Statistical methodology for the concurrent assessment of interrater and intrarater reliability: Using goniometric measurements as an example," Phys. Ther. 74(8), 777-788 (1994)

${ }^{44} \mathrm{~V}$. Chalana and Y. Kim, "A methodology for evaluation of boundary detection algorithms on medical images," IEEE Trans. Med. Imaging 16(5), 642-652 (1997).

${ }^{45}$ B. Chiu, M. Egger, D. J. Spence, G. Parraga, and A. Fenster, "Areapreserving flattening maps of 3D ultrasound carotid arteries images," Med. Image Anal. 12(6), 676-688 (2008).
${ }^{46}$ D. G. Altman and J. M. Bland, "Measurement in medicine: The analysis of method comparison studies," Statistician 32(3), 307-317 (1983).

${ }^{47}$ J. Awad, A. Krasinski, G. Parraga, and A. Fenster, "Texture analysis of carotid artery atherosclerosis from three-dimensional ultrasound images," Med. Phys. 37(4), 1382-1391 (2010).

${ }^{48}$ F. Molinari, W. Liboni, P. Giustetto, E. Pavanelli, S. Giordano, and J. S. Suri, "Use of ultrasound contrast agents in plaque characterization," in Atherosclerosis Disease Management, edited by J. S. Suri, C. Kathuria, and F. Molinari (Springer, London, 2011), pp. 195-219.

${ }^{49}$ D. Adalsteinsson and J. A. Sethian, "A fast level set method for propagating interfaces," J. Comput. Phys. 118, 269-277 (1995).

${ }^{50}$ C. P. Loizou, C. S. Pattichis, M. Pantziaris, T. Tyllis, and A. Nicolaides, "Quality evaluation of ultrasound imaging in the carotid artery based on normalization and speckle reduction filtering," Med. Biol. Eng. Comput. 44(5), 414-26 (2006).

${ }^{51} \mathrm{~F}$. Molinari et al., "Hypothesis validation of far-wall brightness in carotidartery ultrasound for feature-based IMT measurement using a combination of level-set segmentation and registration," IEEE Trans. Instrum. Meas. 61(4), 1054-1063 (2012).

${ }^{52}$ M. Egger, A. Krasinski, B. K. Rutt, A. Fenster, and G. Parraga, "Comparison of B-mode ultrasound, 3-dimensional ultrasound, and magnetic resonance imaging measurements of carotid atherosclerosis," J. Ultras. Med. 27(9), 1321-1334 (2008).

${ }^{53}$ J. R. Mitchell, S. J. Karlik, D. H. Lee, M. Eliasziw, G. P. Rice, and A. Fenster, "The variability of manual and computer assisted quantification of multiple sclerosis lesion volumes," Med. Phys. 23(1), 85-97 (1996).

${ }^{54} \mathrm{P}$. Armitage and G. Berry, Statistical Methods in Medical Research, 2nd ed. (Blackwell, Oxford, 1990), pp. 1-559. 